\title{
Thickness-based adaptive mesh refinement methods for multi-phase flow simulations with thin regions
}

\author{
Xiaodong Chen ${ }^{\mathrm{a}}$, Vigor Yang ${ }^{\mathrm{b}, *}$ \\ a The State Key Laboratory of Nonlinear Mechanics, Institute of Mechanics, Chinese Academy of Sciences, Beijing 100190, China \\ b School of Aerospace Engineering, Georgia Institute of Technology, Atlanta, GA 30332-0150, USA
}

\section{A R T I C L E I N F O}

\section{Article history:}

Received 23 November 2013

Received in revised form 20 February 2014

Accepted 26 February 2014

Available online 5 March 2014

\section{Keywords:}

Thickness-based refinement

Adaptive mesh refinement

Digital topology

Volume-of-fluid

Multi-phase simulation

\begin{abstract}
A B S T R A C T
In numerical simulations of multi-scale, multi-phase flows, grid refinement is required to resolve regions with small scales. A notable example is liquid-jet atomization and subsequent droplet dynamics. It is essential to characterize the detailed flow physics with variable length scales with high fidelity, in order to elucidate the underlying mechanisms. In this paper, two thickness-based mesh refinement schemes are developed based on distance- and topology-oriented criteria for thin regions with confining wall/plane of symmetry and in any situation, respectively. Both techniques are implemented in a general framework with a volume-of-fluid formulation and an adaptive-mesh-refinement capability. The distance-oriented technique compares against a critical value, the ratio of an interfacial cell size to the distance between the mass center of the cell and a reference plane. The topology-oriented technique is developed from digital topology theories to handle more general conditions. The requirement for interfacial mesh refinement can be detected swiftly, without the need of thickness information, equation solving, variable averaging or mesh repairing. The mesh refinement level increases smoothly on demand in thin regions. The schemes have been verified and validated against several benchmark cases to demonstrate their effectiveness and robustness. These include the dynamics of colliding droplets, droplet motions in a microchannel, and atomization of liquid impinging jets. Overall, the thickness-based refinement technique provides highly adaptive meshes for problems with thin regions in an efficient and fully automatic manner.
\end{abstract}

(c) 2014 Elsevier Inc. All rights reserved.

\section{Introduction}

Numerical simulations of multi-scale, multi-phase flows require grid cells sufficiently small to resolve the finest length scale. Examples include liquid jet atomization and droplet breakup. As a consequence of rapid flow evolution, the detailed fluid physics and dynamics can only be resolved by cells several orders of magnitude smaller than the length scale of the fluid at its initial state. The tradeoff between numerical accuracy and cost is a crucial issue from the perspectives of both fundamental science and practical applications. An adaptive mesh refinement (AMR) algorithm is commonly used to efficiently resolve the detailed flow physics in regions with interfacial boundaries. The grid resolution can be adjusted to follow the evolution of the characteristic length scales of flow structures [1,2]. Several studies [3,4] have been conducted using finer meshes with fixed resolution along the interface, to explore the flow dynamics in a thin film formed by approached

\footnotetext{
* Corresponding author.

E-mail address: vigor.yang@aerospace.gatech.edu (V. Yang).
} 
interfaces, for example. It is, however, expensive to use fixed meshes based on the smallest length scale for the entire interface, especially for three-dimensional simulations.

The efficiency of an AMR method can be improved by allowing variable resolution along the interface. For example, local curvature of the interface has generally been used to establish simple refinement criteria [5-9] for an interface along which the curvature varies dramatically. On the other hand, local refinement for thin regions, such as the gas film between two nearby droplets or the liquid sheet formed by impingement of two liquid jets, is still a challenge. For instance, Cristini et al. [9] used two different local length scales based on the curvatures of the interface and gap width between closely spaced interfaces. The local grid density was specified following the local minimum length scale, although the details of the process of obtaining gap width are not discussed in the paper. Mesh splicing operations were required to simulate pinch-off and coalescing events that involve topological changes of interfaces. Nochetto and Walker [10] employed a method capable of detecting imminent topological changes by means of distance functions and shape skeletons. Mesh was adaptively refined until no triangle intersects either the shape skeleton or the interface. This algorithm is complicated and presents challenges with initialization and, for instance, conservation of mass during topological events. Quan [11] implemented a number of length criteria for interface tracking methods with adaptively moving meshes. Following the solution of a set of Laplace equations with the unit normal vectors of interfaces as the boundary conditions, a nominal length scale was introduced for adaptive mesh refinement in thin regions. Although this AMR method functions properly, it involves a complicated algorithm, which slows down simulations and affects accuracy. Moreover, breakup events in small regions cannot be simulated automatically.

In the present work, two thickness-based refinement criteria for multi-scale, multi-phase problems are developed and implemented in a volume-of-fluid (VOF) method, that is, using the Gerris code [8]. The scheme is efficient and robust. The requirement for interfacial mesh refinement can be detected swiftly, without the need of thickness information, equation solving, variable averaging or mesh repairing. The mesh refinement level increases smoothly on demand in thin regions. In this paper, the scheme is verified and validated against several benchmark problems to demonstrate the mesh quality and the overall computational efficiency and robustness. A number of actual physical cases are presented to prove the capability of the technique.

\section{Numerical framework}

\subsection{Governing equations and volume-of-fluid (VOF) method}

The basis of the present study is the general numerical framework of Gerris, documented in Ref. [8]. The formulation accommodates the conservation equations for an incompressible, variable-density flow with surface tension [8]. They can be written in the following vector form:

$$
\begin{aligned}
& \partial_{t} \rho+\nabla \cdot(\rho \boldsymbol{u})=0 \\
& \rho\left(\partial_{t} \boldsymbol{u}+\boldsymbol{u} \cdot \nabla \boldsymbol{u}\right)=-\nabla p+\nabla \cdot(2 \mu \boldsymbol{D})+\sigma \kappa \delta_{s} \boldsymbol{n} \\
& \nabla \cdot \boldsymbol{u}=0
\end{aligned}
$$

where $\boldsymbol{u}=(u, v, w)$ is the velocity vector, $\rho(\boldsymbol{x}, t)$ the fluid density, $\mu(\boldsymbol{x}, t)$ the dynamic viscosity, and $\boldsymbol{D}$ the deformation tensor defined as $D_{i j}=\left(\partial_{i} u_{j}+\partial_{j} u_{i}\right) / 2$. The Dirac delta function $\delta_{s}$ expresses the fact that the surface tension, $\sigma$, is concentrated on the interface. The radius of curvature of the interface is denoted by $\kappa$, and $\boldsymbol{n}$ is the unit outward vector normal to the interface [8].

A volume-of-fluid function $c(\boldsymbol{x}, t)$ is introduced to trace the multi-fluid interface. It is defined as the volume fraction of a given fluid in each cell of the computational mesh. The density and viscosity can thus be written as

$$
\begin{aligned}
& \rho(c) \equiv c \rho_{1}+(1-c) \rho_{2} \\
& \mu(c) \equiv c \mu_{1}+(1-c) \mu_{2}
\end{aligned}
$$

with $\rho_{1}, \rho_{2}$ and $\mu_{1}, \mu_{2}$ being the densities and viscosities of the first and second fluids, respectively.

According to mass continuity, the advection equation for the density takes the following form in terms of the volume fraction:

$$
\partial_{t} c+\nabla \cdot(c \boldsymbol{u})=0
$$

A staggered temporal discretization of the volume-fraction/density and pressure leads to a scheme which is secondorder accurate in time [8]. A classical time-splitting projection method is used, which requires the solution of a Poisson equation. In order to improve numerical efficiency and robustness, the discretized momentum equation is re-organized to a Helmholtz-type equation that can be solved by means of an improved multi-level Poisson solver [8]. The resulting CrankNicholson discretization of the viscous terms is second-order accurate. Spatial discretization is achieved using graded octree partitioning in three dimensions. All the variables are collocated at the center of each discretized cubic volume. Consistent with the finite-volume formulation, variables are interpreted as volume-averaged values for each cell. A piecewise-linear 


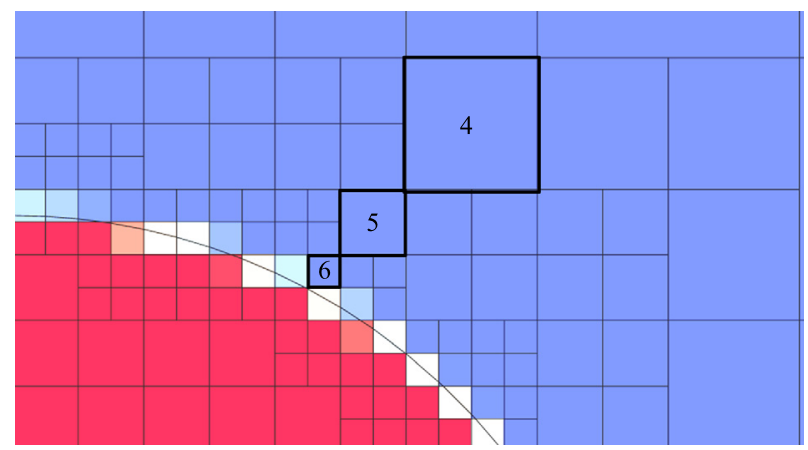

Fig. 1. Quadtree mesh near an interface colored by the volume fraction of each cell.

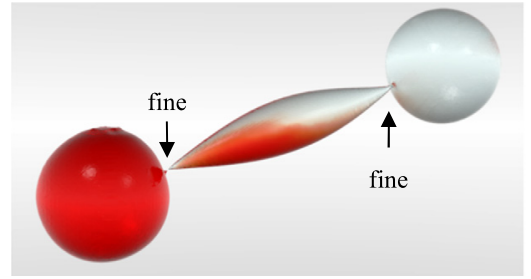

(a)

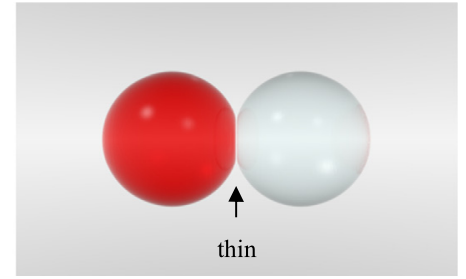

(b)

Fig. 2. Two different types of small region present in droplet collision. (a) Fine ligaments in pinch-off of two ends. (b) Thin gas film between two nearby droplets.

geometrical VOF scheme generalized for quad/octree spatial discretization is used to solve the advection equation for the volume fraction [12]. Since the original Continuum-Surface-Force (CSF) approach [12] suffers from problematic parasitic currents [13], the combination of a balanced-force surface tension discretization and a height-function curvature estimation [8] is used to circumvent the problem. The CFL number is set to be 0.8 for all the simulations to ensure the overall numerical stability.

\subsection{Adaptive mesh refinement}

The above numerical method allows for spatially and temporally varying resolution. In contrast to many previous implementations of mesh adaptation for interfacial flows with Eulerian discretization, the present method is not limited to constant resolution along the interface. In fact, the efficiency of mesh adaptation can be substantially enhanced, particularly when dealing with reconnections and breakup of interfaces. In addition, local mesh refinement or coarsening in quad/octree discretization is cheap and can be performed at every time-step if necessary, with minimal impact on overall performance. Interpolation of quantities on refined or coarsened cells is also relatively simple on regular Cartesian mesh, and is achieved conservatively both for momentum and volume fraction [8]. Several refinement criteria can be employed simultaneously, depending on the physical conditions encountered. The refinement level of the root cell is set to be zero. The level of its children cells is one and so on, recursively. A cell of level $n$ has a resolution of $2^{n}$ in each coordinate. There are two constraints in this adaptive mesh refinement (AMR) method [8]: the levels of direct (sharing line/face) neighboring cells cannot differ by more than one, and the levels of diagonally neighboring cells cannot differ by more than one. These constraints simplify the calculations of the gradient and flux at the cell boundaries [8], and also provide smooth variation of the refinement.

Two AMR schemes, gradient-based (GB) and curvature-based (CB), are commonly used in two-phase flow simulations. The GB criterion for the volume fraction guarantees adequate mesh refinement along the entire interface, where the volume fraction has gradients. Fig. 1 shows an example of quadtree AMR cells near an interface. The cells are colored by the values of the volume fraction in the cell centers. In this case, the GB refinement level of the volume fraction is 6, while level 4 is set to the pure phases. When the length scale changes dramatically across the computational domain, fine meshes are needed in regions with small length scales in order to resolve detailed flow dynamics.

It is, however, costly to apply the GB criterion to all of the interfacial cells based on the minimum length-scale of the fluid flow. To conduct a simulation economically, one may expect to increase the refinement level only in regions with small length scales. The small regions in interfacial flows can be simply classified into two types, fine and thin, quantified by small radius of curvature and thickness, respectively. Fig. 2 shows two different types of small regions during droplet collision. In the pinch-off process of two round ends of the ligament formed by two coalesced droplets, as shown in Fig. 2(a), the diameters of the neck regions (indicated with arrows) change dramatically from the size of the round end to zero. Since the radius of curvature dictates the surface tension, it can be used directly to perform CB refinement to ensure the presence of 


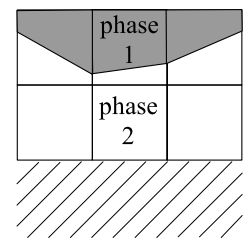

(a)

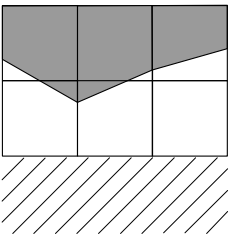

(b)

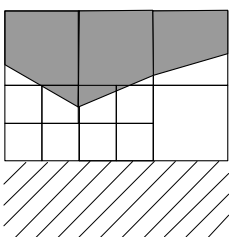

(c)

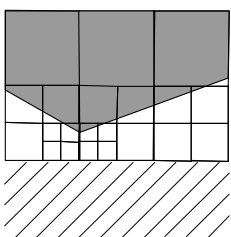

(d)

Fig. 3. Schematic diagrams of thickness-based refinement process near a solid wall. (a) Interface close to the wall with at least one cell in the gap. (b) Interface about to touch wall and rupture. (c) Refinement level of interfacial cells increases to avoid rupture. (d) Refinement levels of interfacial cells further increases to resolve thin region.

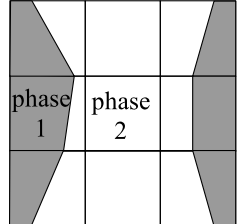

(a)

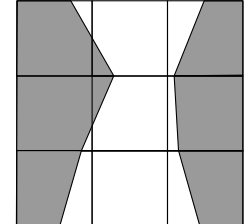

(b)

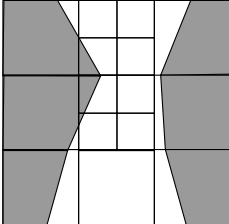

(c)

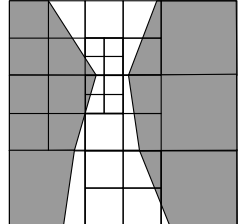

(d)

Fig. 4. Schematic diagrams of thickness-based refinement process for arbitrary condition. (a) Two interfaces separated by at least one cell. (b) Interfaces about to meet. (c) Refinement level of interfacial cells increases to avoid connection. (d) Refinement level of interfacial cells further increases to resolve the nearby interfaces.

sufficient cells in the fine regions [8]. According to the CB refinement criterion, the condition $\Delta x \kappa_{\max }$ is verified within any interfacial cell. Here $\Delta x$ is the cell size and $\kappa_{\max }$ the larger of the two principal curvatures. Cells with $\Delta x \kappa_{\max }$ larger than a prespecified value will be refined to resolve fine curves or surfaces. On the other hand, the thin region between nearby interfaces (Fig. 2(b)) cannot be detected by curvature, where $\kappa_{\max }$ approaches zero. The CB criterion fails, since it is always satisfied. The development of refinement criteria for a thin region is discussed in the following section.

\section{Thickness-based refinement criteria}

To handle a thin region, the characteristic thickness, or length scale, of the region must be identified. The resulting algorithm, however, can be complex and costly. By carefully observing the behaviors of the quad/octree mesh, we can establish thickness-based (TB) refinement techniques. Two different scenarios are considered, as illustrated in Figs. 3 and 4, respectively. The gray and white colors represent two separated fluids, phase 1 and phase 2, respectively. The boundaries between them are represented by the VOF-reconstructed interface. The first case deals with a solid wall or a symmetrical plane (Fig. 3), while the second case involves a thin region surrounded by another fluid (Fig. 4). During numerical simulation, when the gas between two approaching interfaces reaches the smallest cell size, the interface ruptures. The cell size can be used to control the rupture of the interface in an equivalent fashion. In reality, when the interfaces reach a small region in which the van der Waals force takes effect, they will connect quickly under that force. The minimum cell size allowed in simulations should be constrained to accommodate the effect of the van der Waals force.

\subsection{Distance-oriented refinement criterion}

Figs. 3(a) and (b) show the process of an interface approaching a wall in a fixed mesh configuration. In Fig. 3(a), there is one cell of phase 2 between the interface and the wall in each row. When the interface approaches the wall, as shown in Fig. 3(b), the two cells near the wall become interfacial cells, and the interface is about to connect to the wall. Affected by the grid resolution, non-physical rupture of the interface occurs. In order to resolve the flow dynamics in the thin region (film) of phase 2, AMR should be applied to the two under-resolved cells. Fig. 3(c) shows that the interfacial cells near the wall are refined to a higher level. The resultant mesh includes at least one cell of phase 2 located between the interface and wall. As the interface continues approaching the wall, the mesh is further refined, as shown in Fig. 3(d). The proposed process refines specific cells depending on the needs of the problem.

In the TB-AMR process illustrated in Figs. 3(a), (c), and (d), we set at least one cell in the thickness direction. The closer the interfacial cell is to the wall, the higher refinement level it should have. We thus can use the interfacial cells as the reference, instead of the cells inside the thin region, to simplify the refinement process. As presented in Fig. 3(b), once the interface enters the cells next to the wall, the cells of phase 2 become interfacial cells and must be refined. In this case, the distance between the mass center of the interfacial cell and the wall, $d$, varies from zero to one cell size, $\Delta l$, depending on the density ratio of the two phases. This relation can be used to determine whether refinement is needed; if the ratio of $d / \Delta l$ is smaller than unity, the interfacial cell should be refined. Since the levels of neighboring cells cannot differ by more than one (as mentioned in Section 2.2), we can increase the critical ratio to 2 in order to ensure that the interfacial cell is 


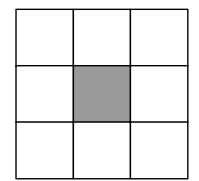

(a)

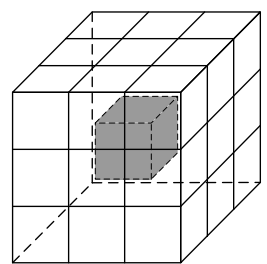

(b)

Fig. 5. Neighboring cells (white) of a target cell (gray) in two-dimensional and three-dimensional domains, involving 8 and 26 neighboring cells, respectively.

one level finer than the cell in the thin region, to obtain good interfacial resolution. If one cell in the thickness direction is not sufficient to achieve expected accuracy, the ratio $d / \Delta l$ can be further increased to allow for a desired number of cells inside the thin region. It should be noted that cells are coarsened only if $d / \Delta l$ is larger than four (prespecified) times the criterion value. If $n$ cells are expected in the thickness direction, a simple criterion becomes

$$
2^{n} \leqslant d / \Delta l \leqslant 2^{n+2}
$$

This distance-oriented (DO) thickness-based criterion is denoted as DO- $n(m)$, where $m$ is the maximum refinement level allowed in the simulation.

At each time-step, the distances of the interfacial cells to the wall are checked, and the interfacial cells that need refinement or coarsening are marked. Since the DO refinement technique is only checked on the interfacial cells, the computational requirements are modest. The DO refinement technique can be applied in any thin regions confined by fixed walls or symmetrical boundaries. Implementation of this criterion is straightforward.

Algorithm 1. Distance-oriented refinement criterion for $n$ cells in the thickness direction, DO- $n(m)$

Step 1. For each interfacial cell, $C$, with a refinement level $L$, if $L<m$,

a. Compute the distance of the mass center of $C$ to the wall, $d$.

b. If $d / \Delta l \leqslant 2^{n}$, mark $C$ for refinement. If $d / \Delta l \leqslant 2^{n+2}$, mark $C$ for coarsening.

Step 2. Process mesh operation.

\subsection{Topology-oriented (TO) refinement criterion}

Figs. 4(a) and 4(b) show the process of two interfaces approaching each other in a fixed mesh configuration. The mesh in Fig. 4(b) is not fine enough to resolve the thin region (film) of phase 2 between the two interfaces. A further approach of the two interfaces causes them to reconnect. Refinement of the cell in the narrowest location allows one cell of phase 2 to exist between the interfaces, as displayed in Fig. 4(c). Fig. 4(d) indicates that finer cells are generated between the interfaces as they approach.

Since the thin region does not have a fixed boundary, the thickness information cannot simply be replaced by the distance from the interfacial cell to the boundary. The TB refinement is designed to prevent topological change caused by grid resolution, and the computational domain itself is representative of the digital domain. We can thus utilize existing digital topology theories to find a refinement criterion.

As one of the fundamental topological properties of a Euclidean space, the Jordan theorem must be applied to establish a local separation rule in Computer-Aided Design (CAD) models [14]. The simple digital topological separation rule states that the vertex-adjacent neighbors (as shown in Fig. 5) of each boundary-intersecting cell must be partitioned in exactly two components: an interior and an exterior. The separation rule can be further simplified to state that every boundaryintersecting cell must have at least one vertex-adjacent interior cell [15]. In numerical simulation, every cell on the interface is surrounded by two different kinds of cells: phase 1 and phase 2, as illustrated in Fig. 1. As long as the topology remains unchanged, this characteristic holds for every interfacial cell. Thus, the topological theory for the interfacial flow requires that every interfacial cell have at least one vertex-adjacent neighboring cell comprising phase 1 , and at least one vertexadjacent neighboring cell comprising phase 2 . If the topology is preserved, the TB refinement can be enforced without utilizing thickness information. Such a topology-oriented criterion can be implemented in an algorithm as follows:

Algorithm 2. Topology-oriented refinement criterion for one cell in the thickness direction

Step 1. For each interfacial cell, $C$, with a refinement level $L$, if $L<m$,

a. Calculate the volume fractions for all the vertex-adjacent neighboring cells.

b. Count the number of cells with the volume fraction of 0 and 1 as $N_{0}$ and $N_{1}$, respectively.

c. If $N_{0}<1$ or $N_{1}<1$, mark $C$ for refinement. If not, mark $C$ for coarsening.

Step 2. Process mesh operation. 
(a) Distance-Oriented (DO)

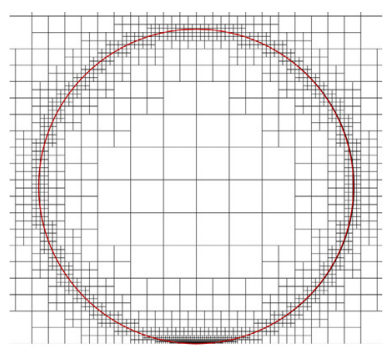

(b) Topology-Oriented (TO)

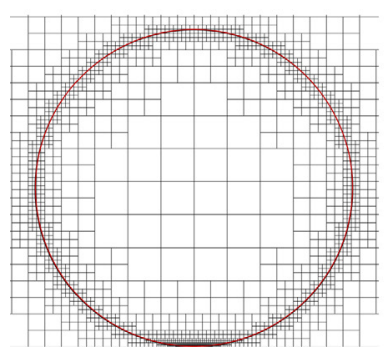

(c) Enlarge view of Distance-Oriented (DO)

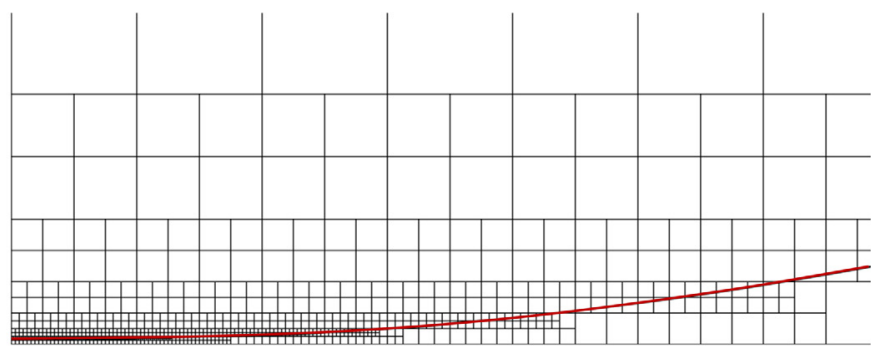

(d) Enlarge view of Topology-Oriented (TO)

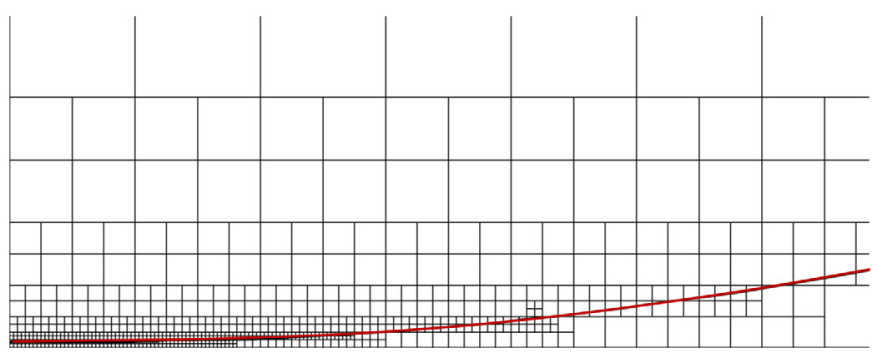

Fig. 6. Meshes for a droplet approaching a wall under different thickness-based refinements. (a) Distance-oriented (DO) refinement. (b) Topology-oriented (TO) refinement. (c) Enlarged view of thin region with DO refinement. (d) Enlarged view of thin region with TO refinement.

\subsection{Extended topology-oriented refinement criterion}

The above refinement criteria are straightforward. The topology of the interface can be preserved to allow at least one cell in a thin region. The interfacial cells and cells within the thin region may be at the same level. Often, only one cell in the thin region is not sufficient to provide accurate results. Finer cells at the interface are always preferred to obtain sharp interfacial resolution. The level of interfacial cell refinement must be one level finer than the cell in the thin region. To meet this requirement, the refinement criterion is improved by taking advantage of the constraint of quad/octree configuration that the levels of neighboring cells cannot differ by more than one.

As the basic refinement criterion in Algorithm 2 checks the vertex-adjacent neighbor of the interfacial cell, it is roughly a topological guarantee to a distance up to the size of the current cell, $\Delta l$. By increasing the checking distance and the size of the checked neighbor cells, the topological guarantee can be extended to ensure any number of cells in the thickness direction. If the expected number of cells in the thickness direction is $n$, virtual neighboring cells of $n$ level lower and with a center-to-center distance of $2^{n} \Delta l$ to the interfacial cell should be checked. This topology-oriented criterion is expressed as TO- $n(m)$. The algorithm proceeds as follows:

Algorithm 3. Topology-oriented refinement criterion for $n$ cells in the thickness direction, TO- $n(m)$

Step 1. For each interfacial cell, $C$, with a refinement level $L$, if $L<m$,

a. Compute the volume fractions for all the virtual cells of level $L-n$ within a distance of $2^{n} \Delta l$ to $C$.

b. Count the number of cells with volume fraction of 0 and 1 as $N_{0}$ and $N_{1}$, respectively.

c. If $N_{0}<1$ or $N_{1}<1$, mark $C$ for refinement. If not, mark $C$ for coarsening.

Step 2. Process mesh operation.

\section{Benchmark examples of thickness-based refinement}

\subsection{Two-dimensional droplet impacts a wall}

As the first example, an inviscid droplet is placed in a constant velocity flow and is moved towards a wall boundary. Fig. 6 shows the situation when the droplet touches the wall. The gradient-based (GB) criterion refines the entire interface to level 8. With the use of the thickness-based (TB), distance-oriented (DO) and topology-oriented (TO) criteria, the mesh is further refined up to level 15, to guarantee a sufficient number of cells in the gap between the droplet and wall. Figs. 6(a) and (b) show the meshes near the droplet at the moment when it touches the wall under the DO-1(15) and TO-1(15) criteria, respectively. The region near the droplet interface is refined extensively. The enlarged views in Figs. 6(c) and (d) indicate that both algorithms can provide at least one cell in the gap near the wall. The refinement results for the two criteria are almost identical. The DO criterion computes the distance from the mass center of an interfacial cell to a fixed 


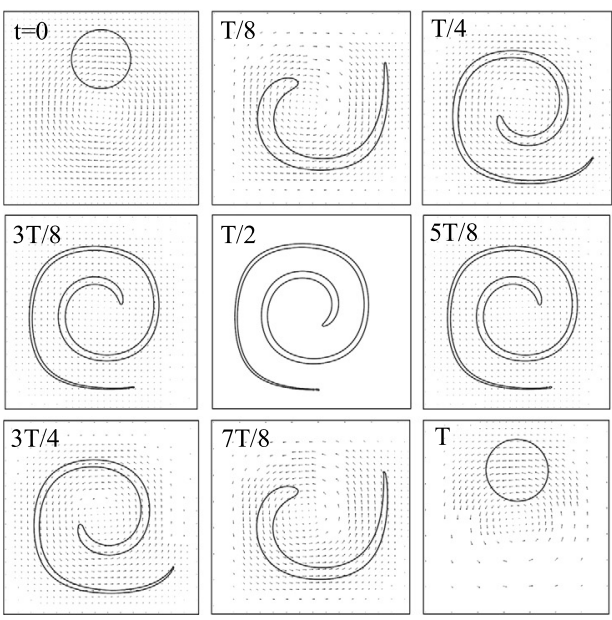

(a)

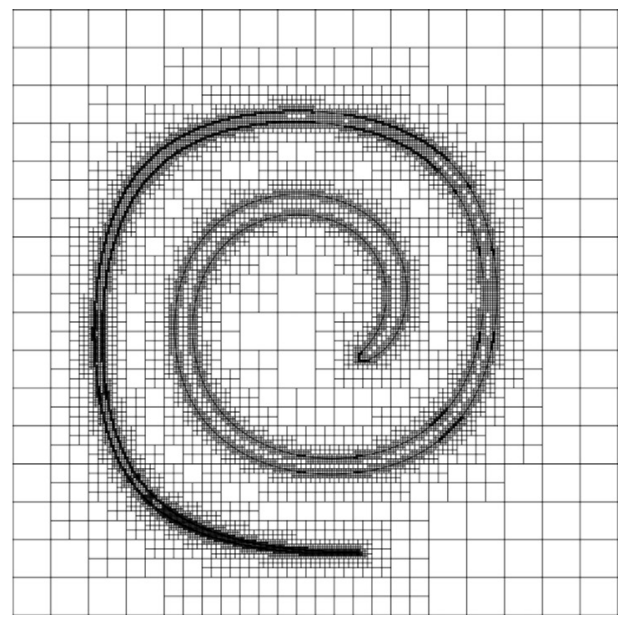

(b)

Fig. 7. Droplet in a time-reversed single vortex flow under topology-oriented refinement TO-2(10). (a) Droplet evolution and velocity vectors. (b) Mesh and droplet surface at the instant with maximum deformation $(t=T / 2)$.

boundary, while the TO criterion computes the values of the volume fractions for the eight virtual cells near the interfacial cell. Although the computational costs are small for both methods, the DO criterion is more efficient than the TO for fixed boundaries.

\subsection{Two-dimensional droplet in time-reversed single vortex flow}

Simple tests of fluid translation and rotation are not sufficient to validate the performance of an interface reconstruction algorithm, since fluid stretching and shearing are also of concern in reality. The test case proposed by Rider and Kothe [16] with an analytical solution of the velocity field [17,18] is performed. A single vortex is set up to stretch a droplet into a spiral with several full rotations, and then reverse the droplet back to its original shape. The straining flowfield is described by the following stream function

$$
\Psi=\frac{1}{\pi} \sin ^{2}(\pi x) \sin ^{2}(\pi y) \cos (\pi t / T)
$$

where $T$ is the time period. The corresponding velocities in the axial and vertical directions are, respectively,

$$
\begin{aligned}
& u=-\frac{\partial \Psi}{\partial y}=-\sin ^{2}(\pi x) \sin (2 \pi y) \cos (\pi t / T) \\
& v=\frac{\partial \Psi}{\partial x}=\sin (2 \pi x) \sin ^{2}(\pi y) \cos (\pi t / T)
\end{aligned}
$$

The maximum deformation occurs at $T / 2$. After one period $T$, the droplet returns to its initial shape. The computational domain is $(1.0 \times 1.0)$, with a circular droplet of diameter 0.3 placed at $(0.5,0.75)$. The time period $T$ is selected to be 8 to demonstrate the capability of the proposed TB refinement method.

Fig. 7(a) shows the droplet evolution from the onset of the deformation to the restoration to the original shape. In this case, GB refinement to level 8 and TO-2 refinement to a maximum level of 10 are applied. Fig. 7(b) shows the mesh with the maximum droplet deformation $(t=T / 2)$. The cell level increases continuously from the head to the tail, to ensure at least two cells in the thickness direction.

Figs. 8(a)-(d) show the droplet shape at the maximum deformation $(t=T / 2)$ based on the gradient-based refinement with four different levels, from $\mathrm{GB}(7)$ to $\mathrm{GB}(10)$, where the quantity within the parentheses stands for the interfacial level. The unphysical rupture of the tail disappears with increasing refinement level. Table 1 shows the cell information for the four cases. Both the total number of cells and the number of cells with the maximum level of resolution increase almost twice with the increase of the interfacial level. Since the tail remains intact at GB(10), this case is considered as a benchmark for the TO refinement.

In order to characterize numerical accuracy and efficiency, four different TO refinement criteria are implemented with $\mathrm{GB}(8)$. The first two cases involve TO-1 with maximum refinement levels of 9 and 10, respectively. The other two cases are TO-2 with maximum refinement levels of 9 and 10, respectively. Figs. 8(e)-(h) show the results. The droplet shapes at the moment of maximum deformation under TO-2 are almost identical to cases to which only GB is applied, with the same maximum level, while results under TO-1 have early breakup in the tail region. 

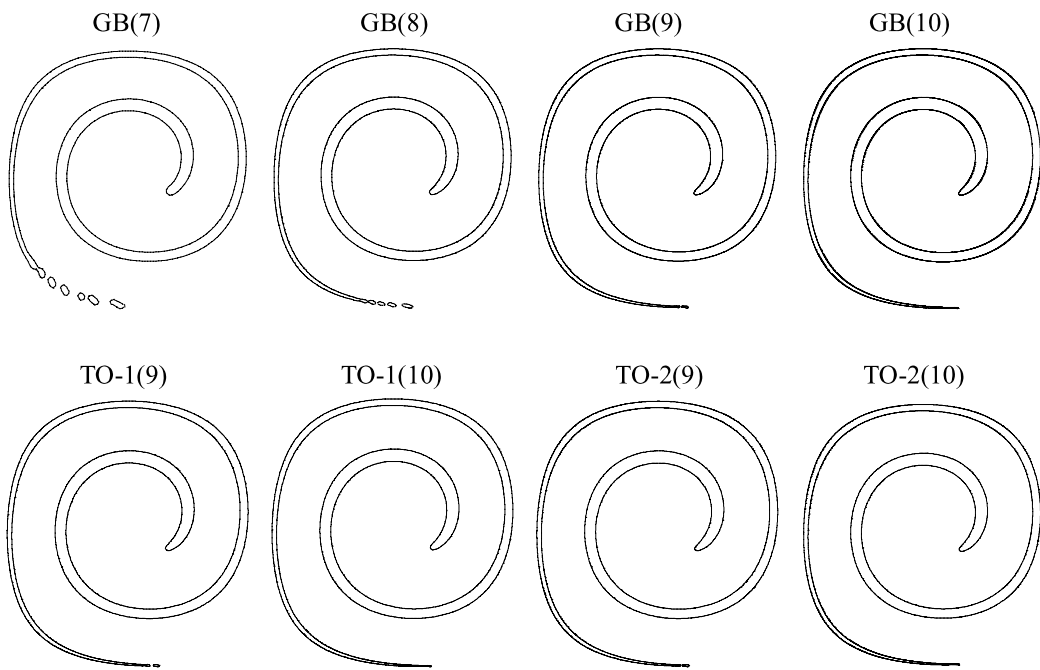

Fig. 8. Interfaces at moment of maximum deformation under different refinement criteria.

Table 1

Cell information for cases with only GB refinement.

\begin{tabular}{llrr}
\hline Level at interface & GB(7) & GB(8) & GB(9) \\
\hline Total number of cells & 5385 & 13005 & 29924 \\
Maximum-level cells & 2896 & 6732 & 64089 \\
\hline
\end{tabular}

Table 2

Cell information for cases under different TO refinement criteria.

\begin{tabular}{lrrr}
\hline Case & TO-1(9) & TO-1(10) & TO-2(9) \\
\hline Total number of cells & 14213 & 15181 & 17657 \\
Level-8 cells & 6523 & 6535 & 6142 \\
Level-9 cells & 1092 & 1181 & 21221 \\
Level-10 cells & - & 604 & 6005 \\
\hline
\end{tabular}

Table 3

$\mathrm{L}_{2}$-norm of differences between the initial and final shapes, and computational time under different refinement criteria.

\begin{tabular}{llllll}
\hline Case & GB(9) & GB(10) & TO-1(9) & TO-2(9) & TO-1(10) \\
\hline $\mathrm{L}_{2}$-norm of error $\left(\times 10^{-2}\right)$ & 4.471 & 3.936 & 4.397 & 4.331 & 3.885 \\
Time $(\mathrm{sec})$ & 322 & 1193 & 146 & 220 & 214 \\
\hline
\end{tabular}

Table 2 summarizes the cell information for the four cases under different TO refinement criteria. Taking the cases under TO-2, for instance, the total cell number increases by only about $20 \%$ from TO-2(9) to TO-2(10). The total cell number of TO-2(10) is 21221 , while the corresponding number of GB(10) is 64089 , or about 3 times larger. The numbers of maximumlevel cells are 2368 and 28336 for the two cases, respectively. The ratio is about $8 \%$.

Table 3 shows the $\mathrm{L}_{2}$-norm of the differences between the initial and final shapes, as well as the computational times for six different cases. All the simulations are performed on a single processor of an Intel ${ }^{\circledR} \mathrm{Xeon}^{\circledR} 2.93 \mathrm{GHz} \mathrm{CPU}$. The computational time for the $\mathrm{GB}(10)$ case is about 3.7 times that under $\mathrm{GB}(9)$. With the same maximum refinement level, the TO-1(9) case takes less than are half of the computational time of $\mathrm{GB}(9)$. On the other hand, the $\mathrm{L}_{2}$-norm of error of TO-1(9) is even smaller than GB(9). The case with TO-2(9) has a little smaller error, but much longer time than TO-1(9). The computational time of TO- $1(10)$ is less than one fifth of that of $\mathrm{GB}(10)$. The $\mathrm{L}_{2}$-norm of error of TO- $1(10)$ is also smaller than $\mathrm{GB}(10)$. As expected, the TO-2(10) case is more accurate, but takes more time than TO-1(9). In spite of its higher maximum refinement level, the TO-2(10) case requires less computational time than GB(9).

It is worth noting from Fig. 7(b) that the TO refinement method can also resolve sharp regions with large curvatures, especially near the head and tail of the spiral shape. To further study this interesting feature, three more cases under TO-1(10), TO-2(10), and TO-3(10), together with GB(4), are simulated. Figs. 9(a), (b), and (c) show the detail meshes in the head region at the moment of maximum deformation. The maximum levels in the head region are 8, 9 and 9 , respectively. At least one, two and three cells are present in the thickness direction, respectively. Figs. 9(d), (e), and (f) show the restored interface with meshes. The cell levels on the interface are 5,6 , and 7 , while $\Delta x \kappa_{\max }$ is $1 / 5,1 / 10$, and $1 / 20$, respectively. 

(a) $\mathrm{TO}-1(10)$
(b) $\mathrm{TO}-2(10)$
(c) $\mathrm{TO}-3(10)$
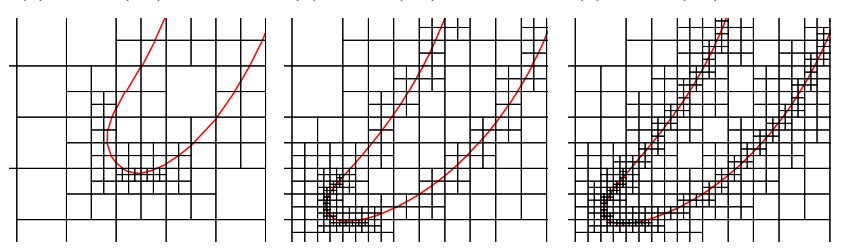

Spiral head end at maximum deformation (d) TO-1(10)

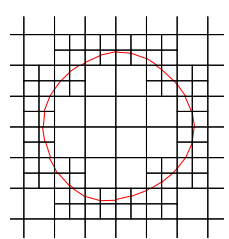

(e) $\mathrm{TO}-2(10)$

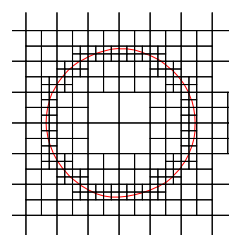

restored droplet (f) $\mathrm{TO}-3(10)$

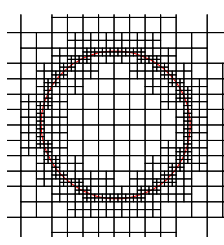

Fig. 9. Detailed meshes for cases with different refinement criteria. (a)-(c) Head regions of the spirals at maximum deformation moment. (d)-(f) Restored droplets.
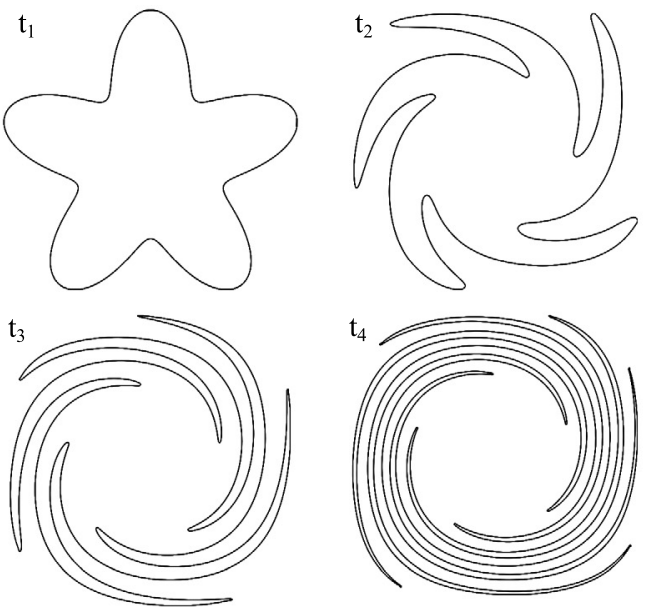

(a)

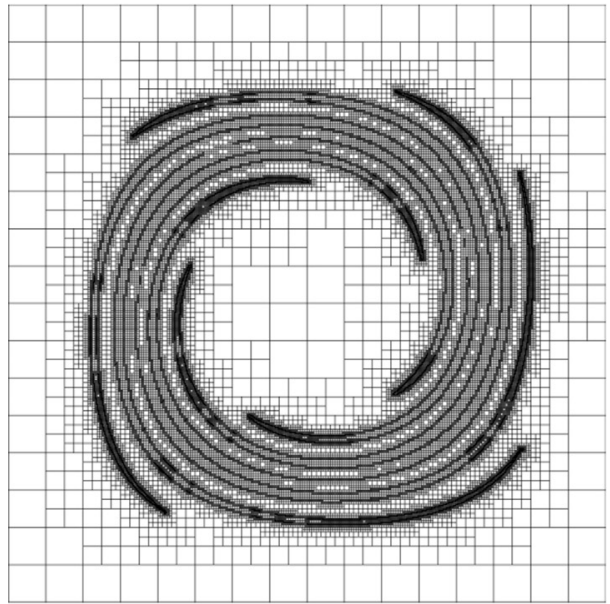

(b)

Fig. 10. Star-shaped interface in a time-reversed single vortex flow. (a) Shape evolution. (b) Mesh and interface at moment of maximum deformation ( $\left.t_{4}\right)$ with TO refinement.

The corresponding resolutions are 5, 10, and 20 cells in the radius direction of the curve, respectively. This indicates that the TO criterion can also act as a CB criterion, and no curvature information is needed. This feature is mainly attributed to the fact that the TO criterion searches neighboring cells in a roughly round pattern.

\subsection{Two-dimensional star-shaped interface in time-reversed single vortex flow}

To test the capability of the TO criterion to deal with multi-layer structures, a two-dimensional star-shape interface in a single vortex flow is modeled with the TO-2(10) refinement. The computational domain is $(1.0 \times 1.0)$ with the center of the star placed at $(0.5,0.5)$, as shown in Fig. 10 . The interface is defined in polar coordinates as

$$
r(\theta)=0.3+0.1 \cos (5 \theta)
$$

The straining flow is defined by the stream function given in Eq. (8).

Fig. 10(a) presents the evolution of the star-shaped interface. As time elapses, the interface deforms and a multi-layer structure forms. Thin regions with phase 1 and 2 appear alternately and are well resolved with TO refinement. Fig. 10(b) displays the mesh and interface at the moment of maximum deformation, $t_{4}$. The thin regions of both phases are well resolved dynamically, with at least two cells in the thickness direction. Without TO refinement, the entire interface in regions with complex geometries must be refined to the finest level throughout the whole process.

\subsection{Three-dimensional droplet in time-reversed single vortex flow}

The above two-dimensional cases demonstrate that TO refinement can detect and resolve a thin region effectively. To validate the capability of the method for three-dimensional problems, a droplet in a time-reversed single vortex flow is considered. LeVeque [18] proposed a three-dimensional flowfield formed by superimposing deformations in both the $x-y$ and $x-z$ planes. The velocities are given by

$$
\begin{aligned}
& u=2 \sin ^{2}(\pi x) \sin (2 \pi y) \sin (2 \pi z) \cos (\pi t / T) \\
& v=-\sin (2 \pi x) \sin ^{2}(\pi y) \sin (2 \pi z) \cos (\pi t / T)
\end{aligned}
$$



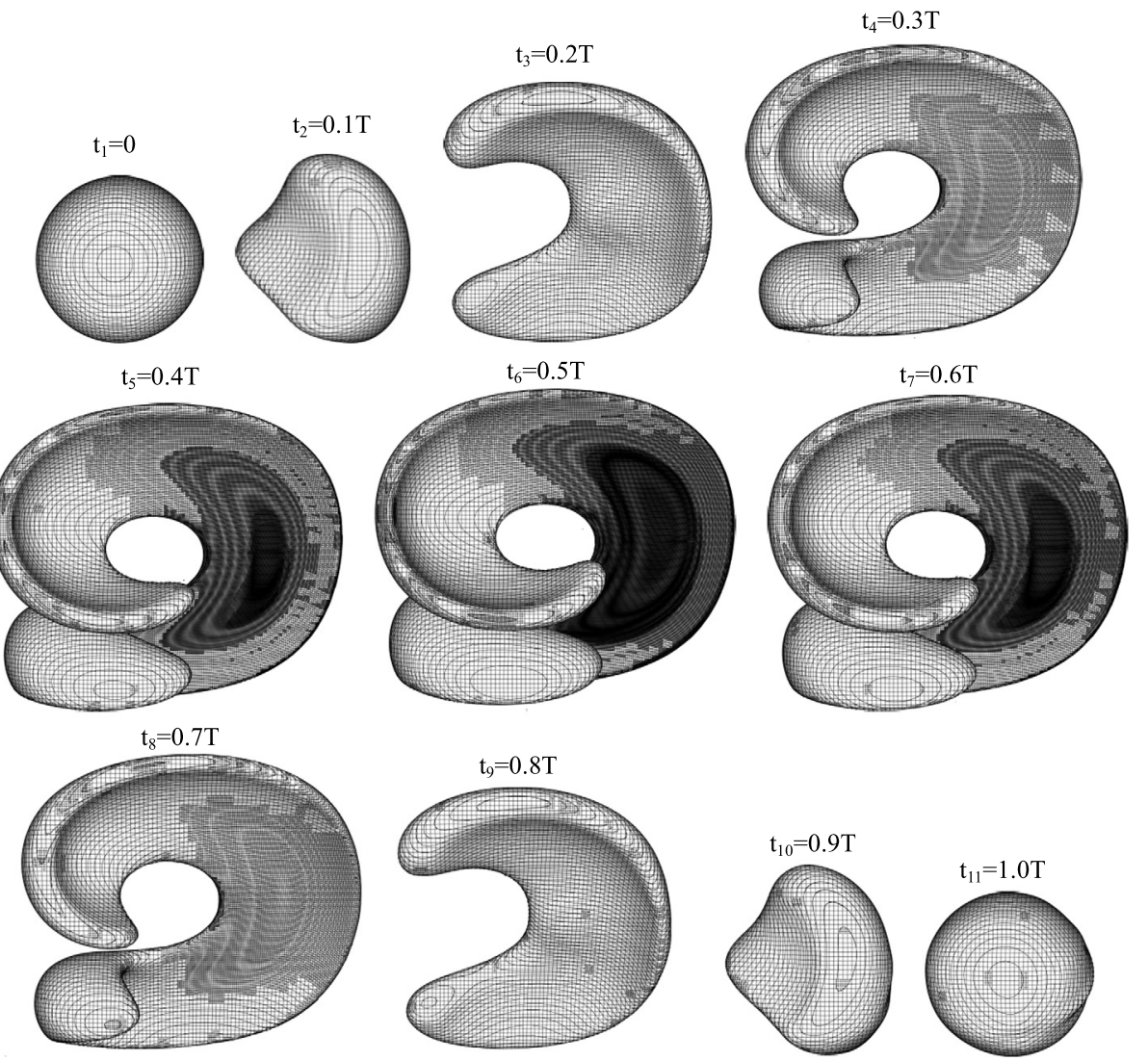

Fig. 11. Shape evolution of a three-dimensional droplet in a time-reversed single vortex flow. The maximum deformation moment is at $t_{6}=0.5 T$.

$$
v^{\prime}=-\sin (2 \pi x) \sin (2 \pi y) \sin ^{2}(\pi z) \cos (\pi t / T)
$$

where $T$ is the time period. The flow reverses at time $T / 2$ and the initial flow condition should be recovered at time $T$. A spherical droplet of diameter 0.3 is placed within a computational domain of $(1.0 \times 1.0 \times 1.0)$. The droplet center is at $(0.35,0.35,0.35)$, and $T=3$.

Fig. 11 shows the evolution of the interfacial mesh for the case with GB(7) and TO-1(9). The number of effective cell is $\left(2^{9}\right)^{3}(=134217728)$. As time proceeds, the droplet deforms and a thin region of varying thickness forms. The cells are refined according to the changing thickness as the flowfield evolves. The maximum deformation occurs at $t=T / 2$. The greatest refinement takes place in the center of the thin region. It is worth noting that the edges with larger curvatures are only partially refined by TO-1(9). The CB refinement feature of the TO criterion does not work well in this three-dimensional case, because any three-dimensional surface has two principal curvatures, $\kappa_{1}$ and $\kappa_{2}$. The TO criterion searches the neighboring cells on a spherical surface and only detects the mean curvature, $\left(\kappa_{1}+\kappa_{2}\right) / 2$, rather than the larger one, $\kappa_{\max }$, to perform $C B$ refinement. The situation in a two-dimensional space is different, since one principal curvature is zero. The TO criterion can thus perform CB refinement. After the maximum deformation, the droplet shape is restored to its initial state. The refinement level decreases as the film thickness increases. Overall, the TO refinement can resolve both spatial and temporal variations of the film thickness.

To check the accuracy and efficiency of the TO refinement for three-dimensional flows, two additional cases with only GB refinement are considered. Fig. 12 compares the calculated interfaces at the maximum deformation and the restored shapes. Figs. 12(a) and (d) show the case with only GB(7). The resolution requires $\left(2^{7}\right)^{3}(=2097152)$ cells for uniform grid. The rupture of the thin film at the maximum deformation and bulges of the restored shape indicate that the level-7 resolution is not sufficient to accurately predict the thinnest regime. Figs. 12(b) and (e) show the case with $\mathrm{GB}(9)$. The smooth interface indicates that the thin film is well resolved at the maximum deformation. Although the restored droplet is still not perfect, the shape is much closer to a sphere than the case with $\mathrm{GB}(7)$ refinement. Figs. 12(c) and (f) show the result with $\mathrm{GB}(7)$ and TO-1(9). The film in the thinnest regime at the maximum deformation is well resolved, as is the case with GB(9), and the restored shape is further improved compared with the case with $\mathrm{GB}(9)$.

Table 4 summarizes the cell numbers for the above cases at the maximum deformation. For the GB refinement, the total number of cells for $\mathrm{GB}(9)$ is about 18 times that of $\mathrm{GB}(7)$. The ratio is approximately the expected value, $4^{2}$, estimated from twice the splitting of interfacial cells from $\mathrm{GB}(7)$ to $\mathrm{GB}(9)$. The total cell number of the case with $\mathrm{TO}-1(9)$ is about $30 \%$ of that with $\mathrm{GB}(9)$, while the ratio of level-9 cells is only about $23 \%$. The computational times of the TO-1(9) and $\mathrm{GB}(9)$ cases 
(a) $\mathrm{GB}(7)$

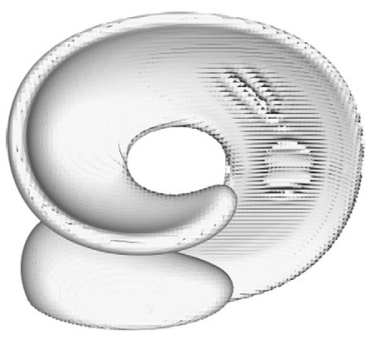

(d) $\mathrm{GB}(7)$

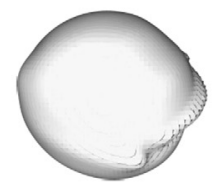

(b) $\mathrm{GB}(9)$

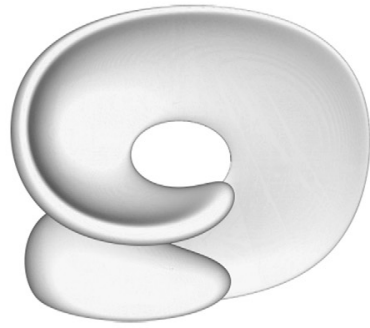

(e) $\mathrm{GB}(9)$

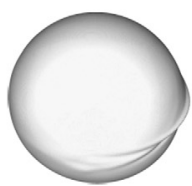

(c) $\mathrm{GB}(7)$ and TO-1(9)

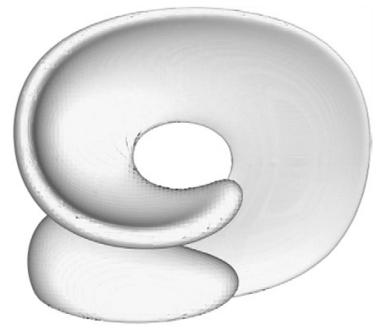

(f) $\mathrm{GB}(7)$ and $\mathrm{TO}-1(9)$

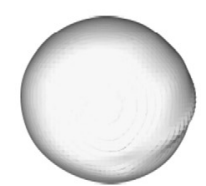

Fig. 12. Three-dimensional droplet in a time-reversed single vortex flow with different refinement criteria. (a)-(c): Maximum deformation. (d)-(f): Restored droplets.

Table 4

Cell numbers for cases with GB and TO refinements at maximum deformation.

\begin{tabular}{llll}
\hline Case & GB(7) & GB(9) & GB(7) and TO-1(9) \\
\hline Total number of cells & 107969 & 1963960 & 596265 \\
Level-7 cells & 72816 & 60438 & 68101 \\
Level-9 cells & - & 1370648 & 311536 \\
\hline
\end{tabular}

are about 79 and 319 minutes, respectively. The difference is a factor of four. The $\mathrm{L}_{2}$-norm of error of $\mathrm{GB}(9)$ is $2.903 \times 10^{-2}$, while that of TO-1(9) is even smaller, $2.350 \times 10^{-2}$.

\section{Demonstration cases}

\subsection{Droplet collision}

Droplet collision plays a fundamental role in the evolution of dense liquid sprays. The collision influences the spray characteristics, including the distributions of droplet size and velocity, local mixing rate, and overall atomization performance. Droplet collision has been studied extensively for decades, both experimentally [19,20] and numerically [21,22]. When two droplets approach each other, the surrounding fluid is trapped between them to form a thin film. High grid resolution is required to capture the detailed flow dynamics in the thin region. The TB refinement technique developed in the present work provides an efficient means to resolve the underlying physics affected by the thin gas film. Two different types of bouncing (head-on and off-center) of equal-sized droplets are presented in this section, simulating the experiment of Qian and Law [23].

\subsubsection{Bouncing of head-on droplet collision}

For head-on droplet collision, the impact plane is a symmetrical plane of the flow field. Thus, DO refinement with respect to the impact plane is used to perform local refinement in the thin region. For demonstration purposes, we consider an axisymmetric model. The physical model involves two tetradecane droplets in a nitrogen environment at 1 atm pressure. The density and viscosity ratios are about 666 and 119, respectively. The droplet size is $306 \mu \mathrm{m}$. The Weber and Reynolds numbers are 8.6 and 105.9, respectively, defined as We $\equiv \rho U^{2} D / \sigma$ and $\operatorname{Re} \equiv \rho U D / \mu$. Here, $\rho$ is the liquid density, $U$ the relative velocity of approaching droplets, $D$ the droplet diameter, $\sigma$ the surface tension, and $\mu$ the liquid viscosity. Cells with refinement level 8 are set for both the interface and the interior of the droplets. Refinement DO-2(15) is employed to resolve the thin gas film between the droplets.

Fig. 13 shows the droplet evolution with realistically rendered images created using the ray-tracing technique. A dye variable is used in the simulation to trace mass from different droplets; it is displayed in red with volume rendering. Increasing the cell refinement level according to the thickness of the gas film permits good resolution of the dynamics in the thin region, even under large deformation of the droplets. At $t_{1}$, the droplets are approaching each other. A flat gas film is formed at $t_{2}$ to prevent the merging of the nearby interfaces. The diameter of the gas film increases through $t_{3}$ and $t_{5}$, while the inertia flattens the spherical droplet. During this process, the droplet kinetic energy is transferred to the surface energy due to increasing surface area. After the maximum deformation is achieved at $t_{5}$, droplets begin to restore their 

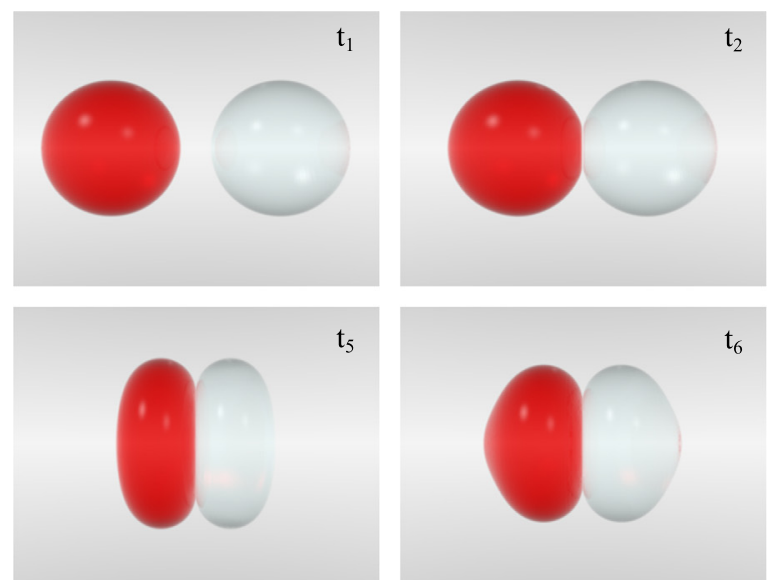

$\mathrm{t}_{2}$

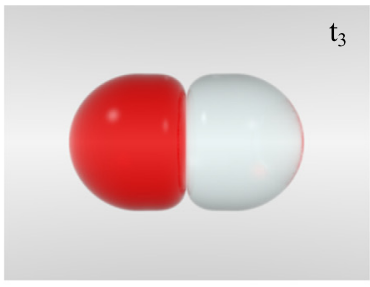

$t_{6}$

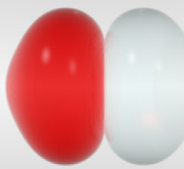

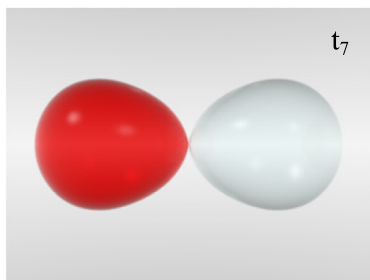

$\mathrm{t}_{7}$
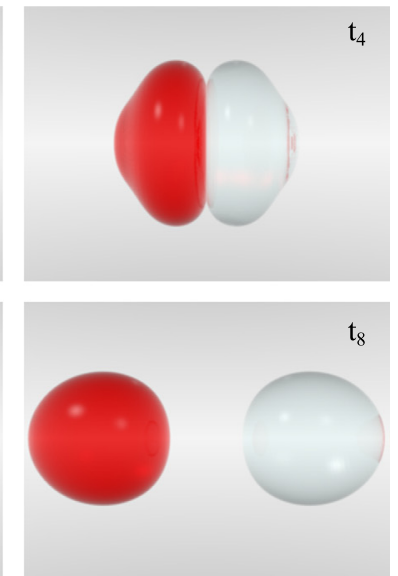

Fig. 13. Shape evolution of head-on droplet collision with bouncing outcome. Tetradecane droplets in nitrogen at $1 \mathrm{~atm}$, We $=8.6$, Re $=105.9, D=306 \mu \mathrm{m}$, $U=0.97 \mathrm{~m} / \mathrm{s}$. (For interpretation of the references to color in this figure legend, the reader is referred to the web version of this article.)

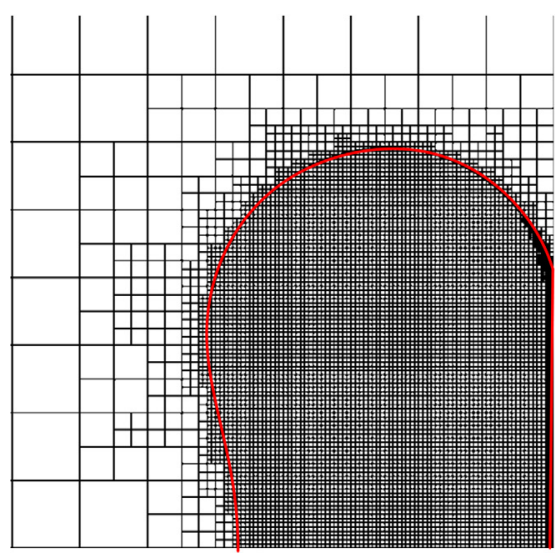

(a)

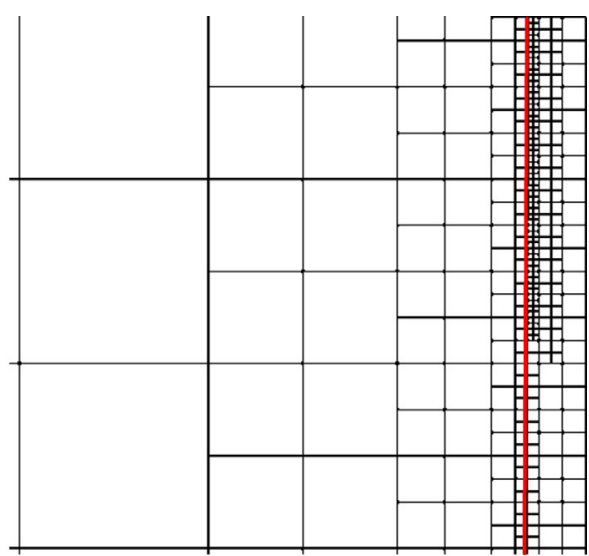

(b)

Fig. 14. (a) Axisymmetric mesh used in the simulation of the left-approaching droplet at $t_{5}$ of Fig. 13. (b) Enlarged view near the impact plane showing the minimum cell in the simulation (256 times enlargement of the left figure).

spherical shape under surface tension. Energy is then transferred from the surface to kinetic form, with increased droplet velocities. Eventually, the droplets separate and finish the bouncing process.

Fig. 14 shows detailed meshes and interfaces of the left-approaching droplet at the maximum deformation $\left(t=t_{5}\right)$. Fig. 14(a) is a part of the axi-symmetric mesh used in the simulation. The bottom line is the axis of symmetry, while the right boundary is the symmetrical surface representing the impact plane. Fine cells are generated near the impact plane with the implementation of the DO-2(15) refinement technique. Fig. 14(b) is an enlarged view of the thin region between droplets. The smallest cell is at level 15 , with a size of about $5 \times 10^{-5} \mathrm{D}$ (about $0.015 \mu \mathrm{m}$ ). The calculated minimum thickness of the gas film is about $0.3 \mu \mathrm{m}$. As the minimum thickness is much larger than the dominating distance of the van der Waals force (about $0.03 \mu \mathrm{m}$ ), the real physical process of bouncing is resolved in the hydrodynamic range. The grid resolution increases smoothly to guarantee at least two cells in every thickness direction. The AMR technique established here is show to be robust and efficient; the refinement is effectively applied at every time step to assure temporal and spatial accuracy.

\subsubsection{Bouncing of off-center droplet collision}

This case was studied using a three-dimensional model with the TO refinement technique. The Weber number is We $=$ 48.8 , and the off-center distance/diameter ratio is $B=0.9$. The physical properties of the fluids are identical to those of the head-on case discussed in Section 5.1.1. Both GB(8) and TO-2(11) refinements are employed, with a minimum cell size of about $0.7 \mu \mathrm{m}$. Fig. 15 shows the droplet evolution with realistic rendering. At $t=t_{1}$, two droplets approach each other with a small overlap region in the vertical direction. A gas film forms at $t_{2}$ due to the stagnation flow and deformable interfaces. Positive pressure inside the gas film prevents merging of the interfaces. Images at $t_{2}-t_{7}$ show the evolution of the gas film, from its formation to disappearance. Eventually, the two droplets bounce apart and move in their original directions without 

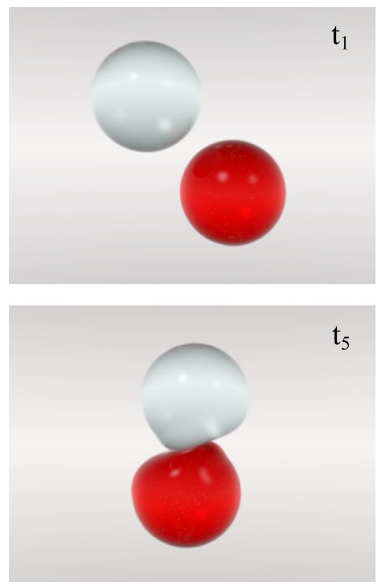
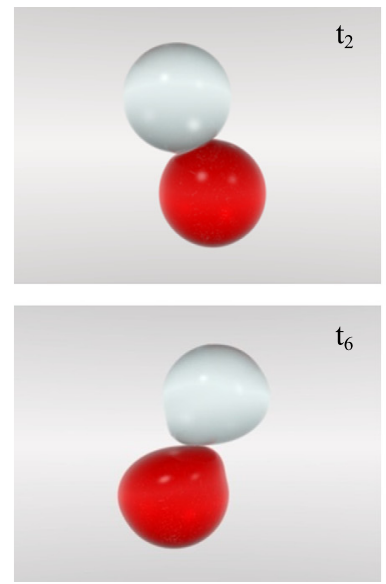
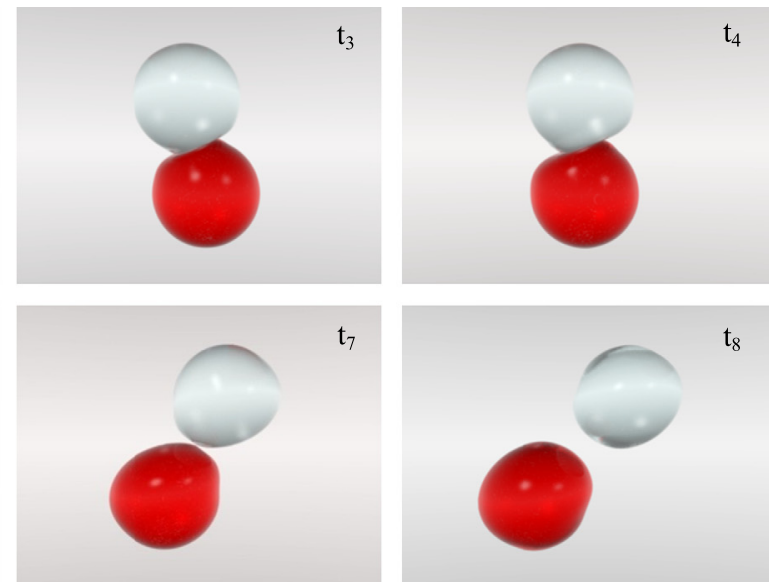

Fig. 15. Shape evolution of off-center droplet collision with bouncing outcome. Tetradecane droplets in nitrogen at 1 atm, We $=48.8$, Re $=260.3, B=0.9$, $D=306 \mu \mathrm{m}, U=2.31 \mathrm{~m} / \mathrm{s}$.
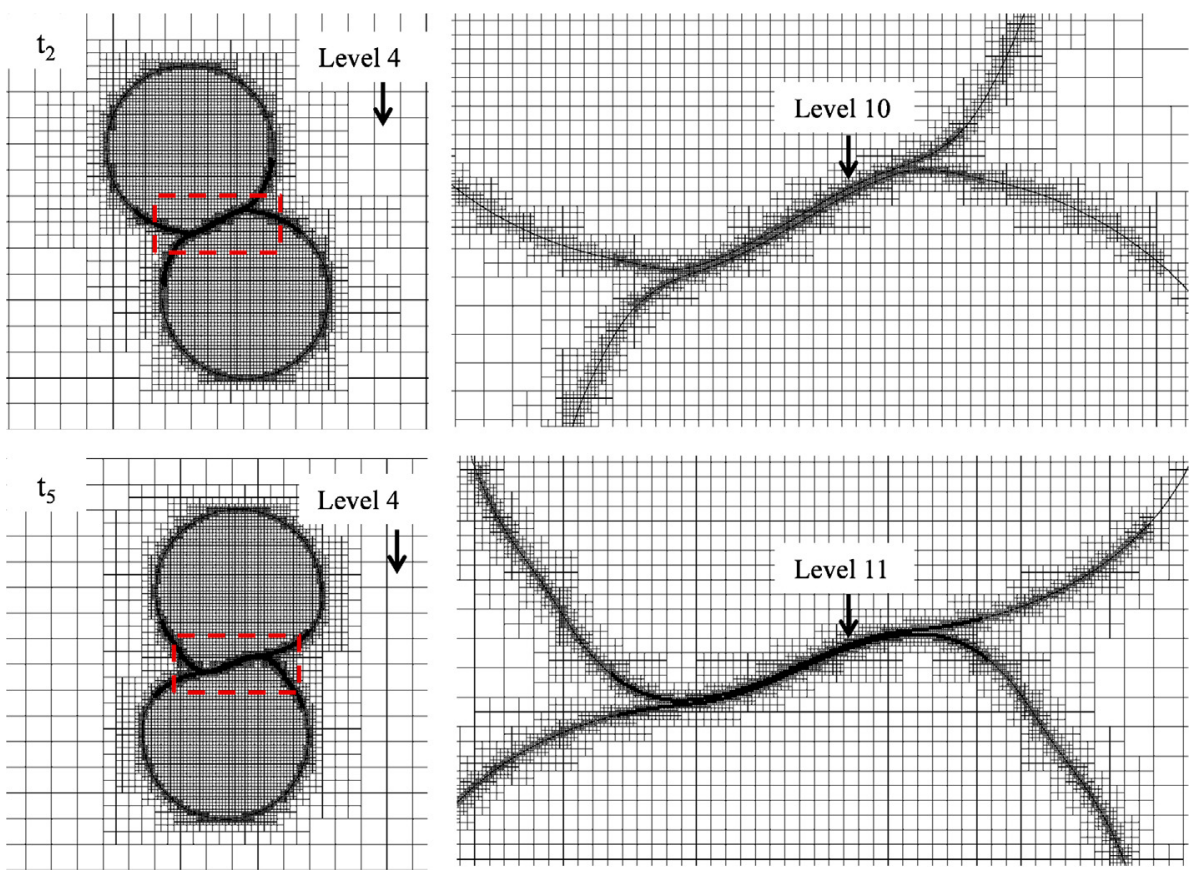

Fig. 16. Meshes at $t_{2}$ and $t_{5}$ during off-center bouncing of droplets shown in Fig. 15. Left: meshes near the droplet surface. Right: enlarged views near the gas film.

any contact. The droplet dynamics, especially the gas-film behaviors, are resolved in detail. The experimental observation discussed in Ref. [23] is faithfully simulated.

Fig. 16 shows the meshes in the cross section cut through the droplet center at two time instants, $t_{2}$ and $t_{5}$. The cells with higher levels are located in the region of the gas film. At $t_{2}$, the maximum refinement level is 10, as shown in Fig. 16(a). When the thickness of the gas film decreases under the droplet motions perpendicular to the film, the refinement level increases to 11 at $t_{5}$, as shown in Fig. 16(b). The thickness-based refinement functions well, both locally and dynamically, resulting in a significant saving of computational time, especially for three-dimensional simulations.

\subsection{Droplet-based microfluidics}

Droplet-based microfluidics involves flow dynamics in thin liquid films between droplets and a confining wall or between approaching droplets. The thin region, however, is either not well resolved or simply avoided in existing numerical techniques. A constant contact angle close to $180^{\circ}$ [24] or a non-wetting [25,26] condition is commonly used to prevent the droplet from contacting the channel wall or obstacle. The disjoining pressure in a thin liquid film is neglected. In this sec- 
(a) initial mesh and interface

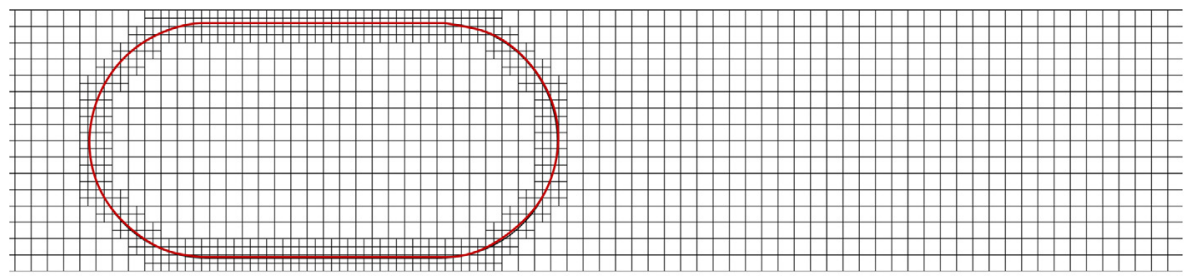

(b) meshes near confined wall
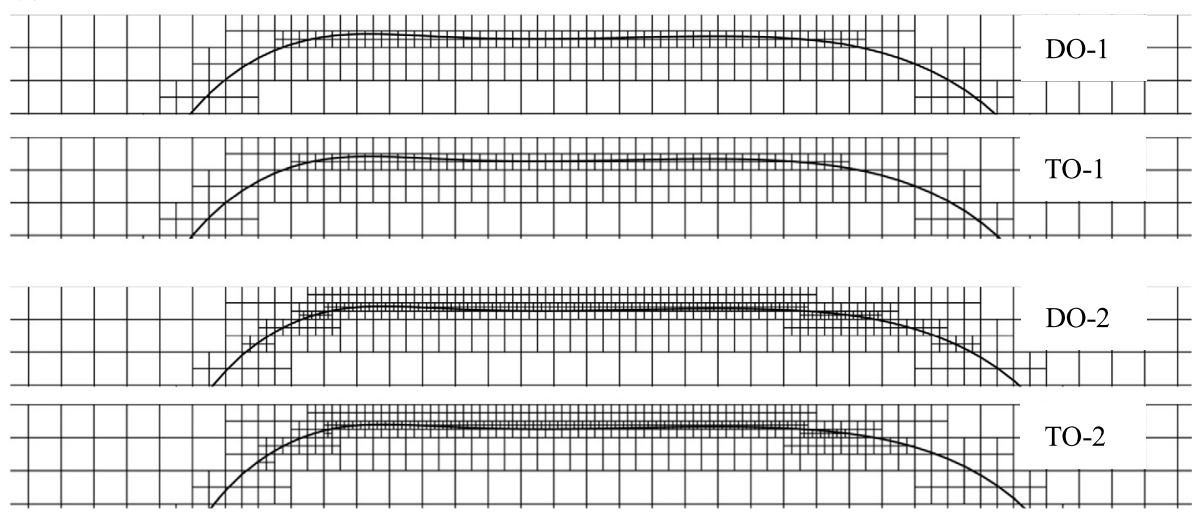

Fig. 17. Meshes of two-dimensional droplet moving in microchannel. (a) Initial meshes and interface. (b) Enlarged meshes near confined wall.

tion, several microfluidic cases are presented to demonstrate the capabilities of the AMR method developed in the present study.

\subsubsection{Two-dimensional droplet moving in a microchannel}

A two-dimensional water droplet moving in a microchannel filled with oil, as shown in Fig. 17, is simulated using different TB criteria. The dispersed phase is Milli- $Q$ water and the continuous oil phase is hexadecane [27]. A low surface tension, $5 \mathrm{mN} / \mathrm{m}$, is set for the Milli-Q water, due to the addition of surfactant. The channel is a $23 \mu \mathrm{m}$ wide microchannel. The ratio of the initial length of the droplet to the height of the microchannel is two. The Weber and Reynolds numbers based on the water properties and the channel height are 0.50 and 0.0031 , respectively. Initially, a parabolic velocity distribution with a mean value of $0.025 \mathrm{~m} / \mathrm{s}$ is set for the entire computational domain. A no-slip condition is enforced on the walls. The GB refinement is applied to refine the interface to level 5, while cells of level 4 are used for the rest of the computational domain.

Fig. 17(a) shows the initial mesh of the simulation. Four cases with different TB criteria are carried out. Figs. 17(b) and (c) show the meshes at the same physical time when the flow reaches a steady state. Both the DO- 1 and TO- 1 refinements are able to resolve the thin region, with at least one cell in any location. The overall interfacial meshes are indistinguishable, except for the cells at the ends of the refined thin regions. For the DO-2 and TO-2 refinement, at least two cells exist in any thickness direction. All the cases exhibit nonmonotonic variations of the film thickness along the flow direction. The maximum thickness is located near the center of the film, because of the higher pressure inside the film caused by the lubrication effect. The film dynamics is captured efficiently by the TB refinement.

\subsubsection{Two-dimensional droplet interaction in an expanding channel}

Droplet interaction in a microchannel is an important topic in droplet-based microfluidics. When the droplets approach each other, a thin film between them forms to avoid connection of the interfaces. Such a thin film may persist for a long time, and fine cells are needed to resolve the internal flow dynamics. The film thickness may be $O\left(10^{-4}\right)$ of the droplet diameter before rupture under the effect of the van der Waals force. The TO refinement is robust enough to solve this kind of problem over a wide range of length scales and time periods.

Following the experiments of Bremond et al. [27], we consider a droplet pair moving in an expanding microchannel, as shown in Fig. 18. The fluid properties are identical to those in the previous section. The channel height starts at $36 \mu \mathrm{m}$ and expands to $72 \mu \mathrm{m}$. The mean flow velocity is $0.056 \mathrm{~m} / \mathrm{s}$. The ratio of the initial length and height of the droplet is 3.13 . The Weber and Reynolds numbers based on the water properties and the channel height are 1.78 and 0.0020 , respectively. GB refinement is applied to refine the interface to level 6, while cells of level 3 are used for the rest of the region. The TO-2(10) is used with a minimum cell size of about $0.02 \mu \mathrm{m}$, approximately the effective distance of the van der Waals force.

Figs. 18 shows the dynamics of the approaching droplets. With the use of the TO-2 refinement, the thin regions both near the walls and between the two droplets are resolved efficiently. When the right droplet moves into the expanding part 

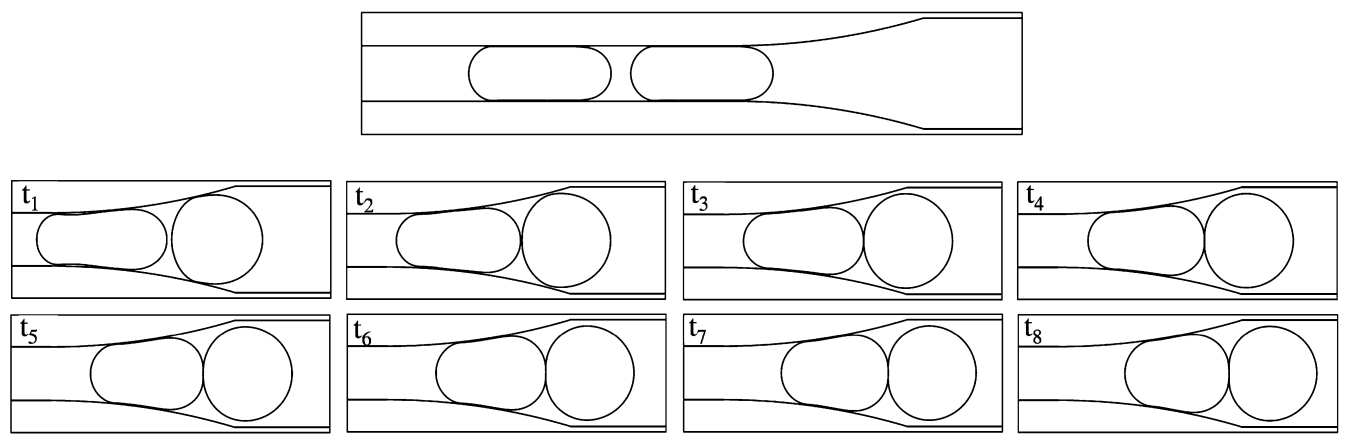

Fig. 18. Shape evolution of two-dimensional droplet interaction in an expanding channel. Top: first droplet enters the expanding channel. $t_{1}-t_{8}$ : thin film forms and moves downstream with the droplets in the channel.
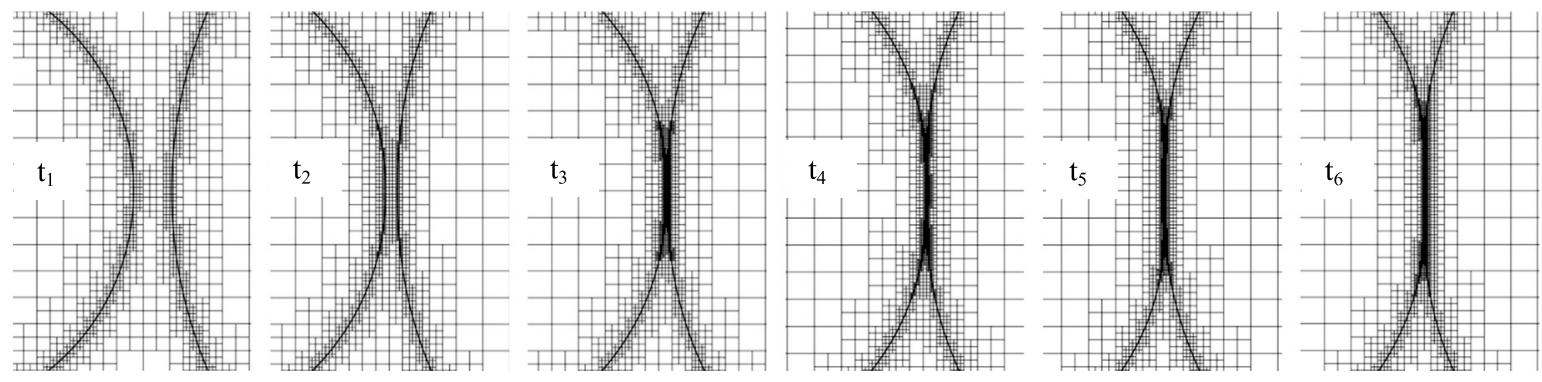

Fig. 19. Enlarged views of interface and meshes near the thin film formed by approaching droplets in a microchannel.

of the channel, it decelerates and expands laterally with the increasing height of the channel. The oil between the droplets is drained out quickly from $t_{1}$ to $t_{2}$. The thin film between the droplets then prevents the connection of droplets, while they move together downstream.

Fig. 19 presents the evolution of the meshes near the film formed between the two droplets. The TO-2 refinement does not take effect if there are more than two cells between the droplets, as shown at $t=t_{1}$. When the droplets are closer to each other at $t=t_{2}$, the interfacial areas are refined under the action of TO-2. The resolution in the film region continues to increase to avoid rupture as the two droplets continue to approach. After the thin film forms, the rate at which the droplets change shape decreases, due to the lubrication effect. The flowfield inside the film becomes nearly stagnant. Rupture occurs when the film thickness reaches the smallest cell size.

\subsubsection{Three-dimensional droplet moving in a microchannel}

Three-dimensional simulations are required to explore more detailed flow physics. The TB refinement is applied only in regions of concern, to minimize the computational cost. We first consider a single droplet moving in a microchannel, as shown in Fig. 20. Two cases are treated with different confinement conditions. The fluid properties and the geometry parameters are identical to those in Section 5.2.1. Since the DO and TO criteria are almost indistinguishable in the near-wall region, only the DO-2 refinement is applied to capture the flow dynamics in regions between the droplet and walls.

For the first case, the ratio of the channel width and height is set to three. The droplet has an equivalent diameter larger than the height but smaller than the width of the channel. The droplet is confined by the top and bottom walls, but is located at a slight distance from the side walls. The droplet initially has a cheese-wheel-like shape. Fig. 20 shows the meshes when the simulation reaches its steady state. Fig. 20(a) shows the VOF-reconstructed interface with the edges of interfacial cells. Since the wall is fixed, the low velocity of the fluid in the film provides a cushion effect, which separates the interface from the wall. Thin regions form near the top and bottom walls. Fig. 20(b) shows a section across the center of the droplet. The refinement level varies as required to resolve the film thickness. The spatial distribution of the film has a concave shape. Figs. 20(c) and (d) show the top and side views of the droplet surface with cells. Refined interfacial cells at a given level show a circular pattern. The DO-2 refinement is capable of adapting meshes based on the evolution of the film. The detailed droplet configuration is faithfully simulated with proper resolution.

In the second case, the equivalent diameter of the droplet is smaller than both the depth and width of the microchannel, which have the same value. The droplet is confined by all the walls. The initial shape of the droplet is set to be ellipsoid. Fig. 21(a) shows the VOF-reconstructed interface with edges of interfacial cells. Thin regions form near the top, bottom, left and right walls. Fig. 21(b) shows a cross section through the droplet center in the streamwise direction. Unlike the previous case, the film thickness increases toward the leading surface of the droplet. Figs. 21(c) and (d) show the top and side views of the droplet surface with cells. Identical meshes are obtained on both sides, with interfacial cells of the same levels distributed in the elliptical regions. 
(a)
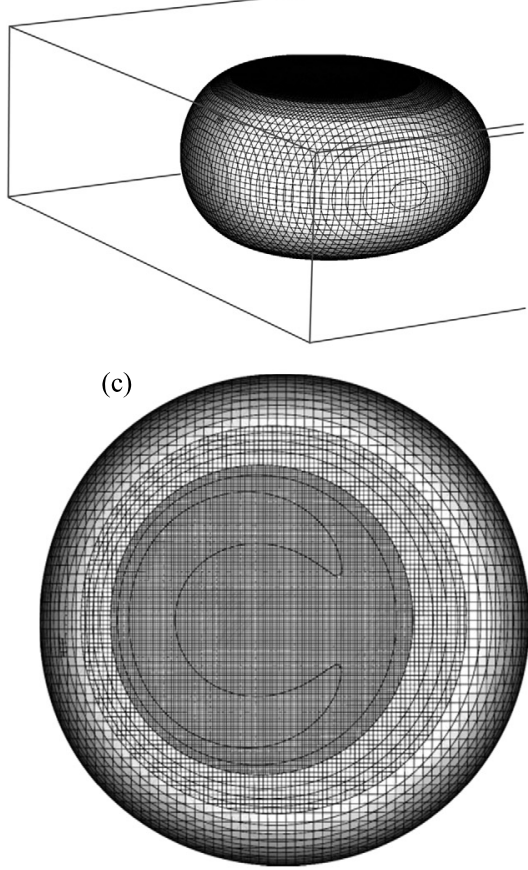

(b)

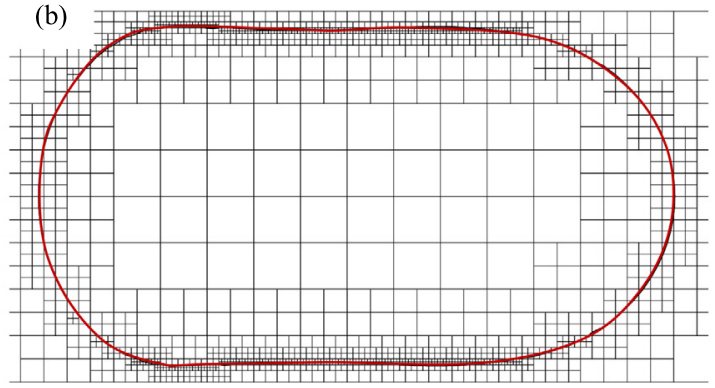

(d)

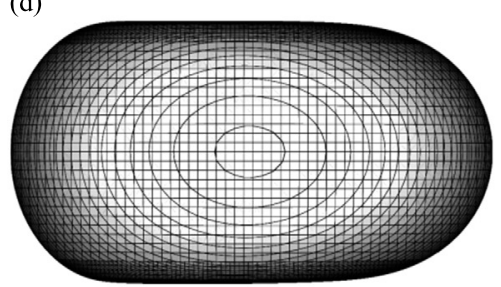

Fig. 20. Droplet in a microchannel with a width/height ratio of 3. The droplet surface is represented using VOF-reconstructed planar fragments in each interfacial cell. (a) Perspective view. (b) Mesh in the cross section. (c) Top view. (d) Side view.

(a)

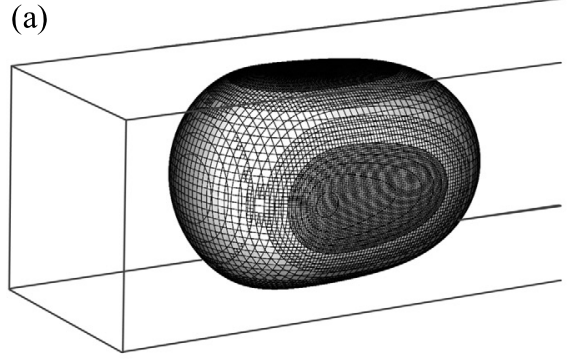

(c)

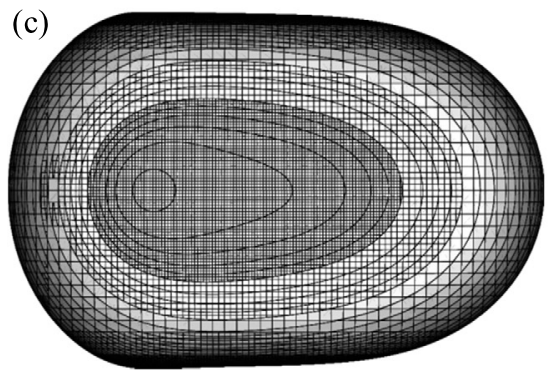

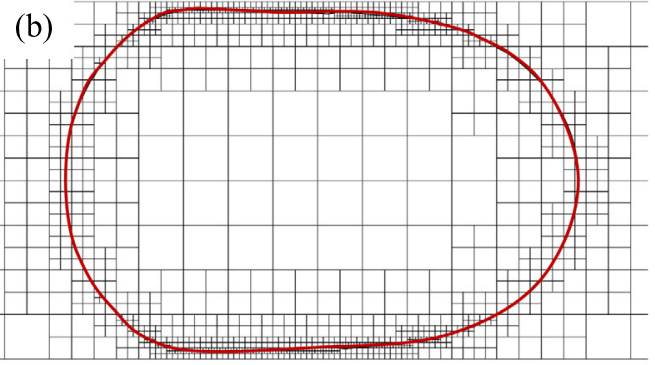

(d)

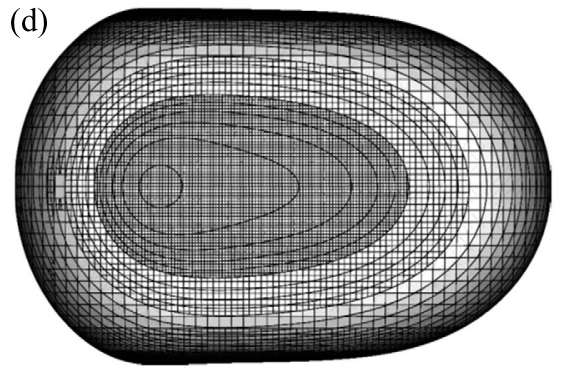

Fig. 21. Meshes of three-dimensional droplet in a microchannel with a width/height ratio of 1 . The interface is represented using VOF-reconstructed planar fragments in each interfacial cell. (a) Perspective view. (b) Meshes in the cross section. (c) Top view. (d) Side view.

\subsection{Impinging jet atomization}

The present TO refinement technique has also been employed in numerical simulations of liquid-jet atomization [28]. When two liquid jets impinge on each other, a sheet is established at the impact point, along with the formation of a rim around the sheet under the effect of surface tension. Both fine and thin regions exist in this complex flow process. The $\mathrm{CB}$ and TO-2 refinements are used in combination to ensure proper resolution throughout the entire field. Fig. 22(a) shows the impingement of two liquid jets with different velocities and the ensuing fish-bone-like pattern of the sheet. The grid resolution is adapted in accordance with the variation of local length scales. Fig. 22(b) shows an enlarged view of interfacial 


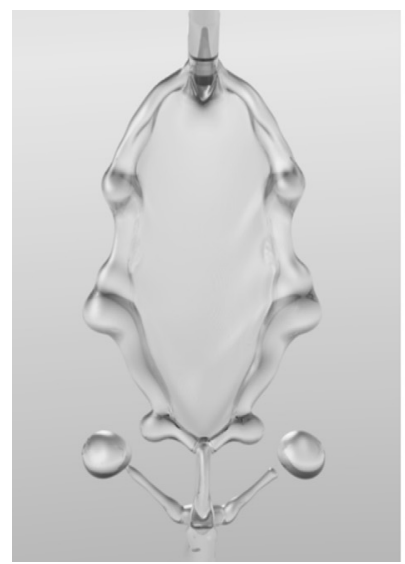

(a)

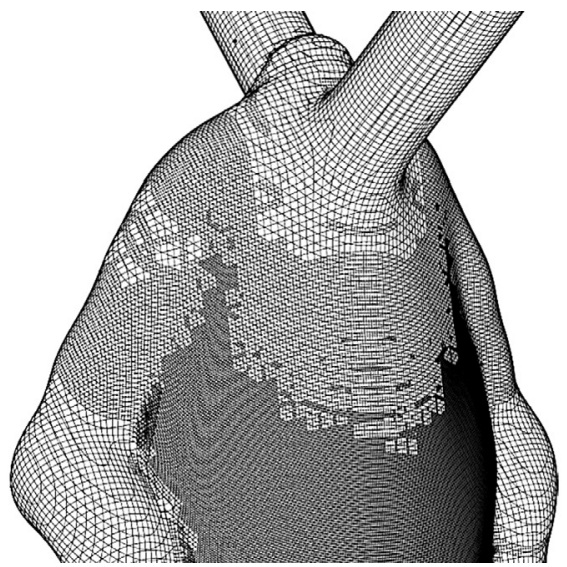

(b)

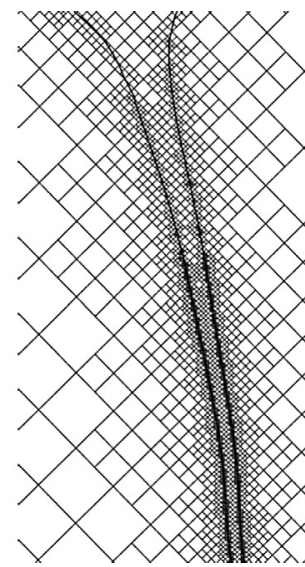

(c)

Fig. 22. Interface and meshes of impinging jet. The interface is represented using VOF-reconstructed planar fragments in each interfacial cell. (a) Overall flow structure. (b) Interface with interfacial cells near the impact point. (c) Meshes in the cross-section of the liquid sheet.

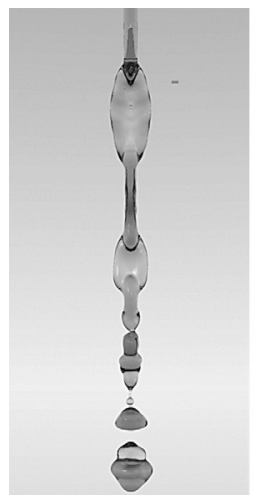

(a)

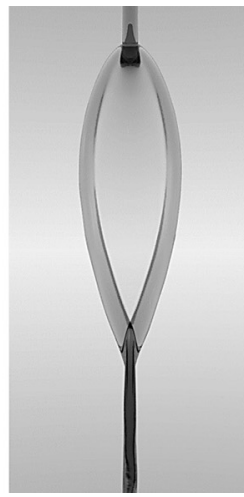

(b)

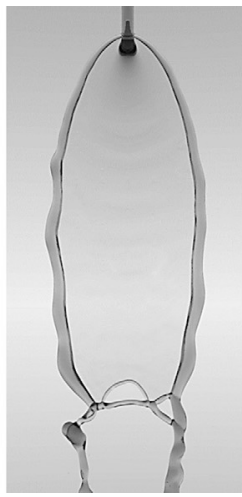

(c)

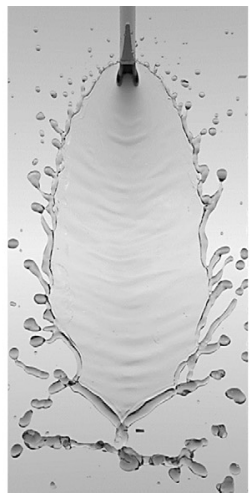

(d)

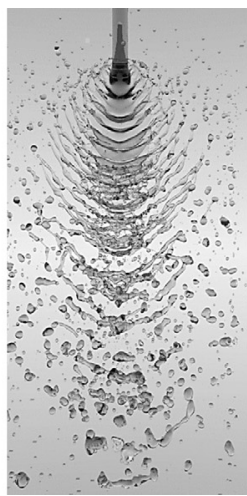

(e)

Fig. 23. Impinging-jet flow patterns of glycerin-water solution obtained from simulations (movie showing flow development can be found in Chen and Yang [29]). $D=400 \mu \mathrm{m}, 2 \alpha=60^{\circ}$, (a) liquid chain $\left(u_{j}=2.2 \mathrm{~m} / \mathrm{s}\right.$, We $=27.5$, Re =1000); (b) closed rim $\left(u_{j}=3.3 \mathrm{~m} / \mathrm{s}, \mathrm{We}=58.8, \operatorname{Re}=40.4\right)$; (c) disintegrating sheet $\left(u_{j}=5.3 \mathrm{~m} / \mathrm{s}\right.$, We $=152$, Re $\left.=294\right)$; (d) unstable $\operatorname{rim}\left(u_{j}=7.9 \mathrm{~m} / \mathrm{s}, \mathrm{We}=343.5, \operatorname{Re}=3536\right) ;(\mathrm{e})$ impact wave $\left(u_{j}=11.2 \mathrm{~m} / \mathrm{s}, \mathrm{We}=687, \operatorname{Re}=5000\right)$.

cells near the impact point. The cell refinement level increases with decreasing film thickness in the downstream region. Fig. 22(c) presents the meshes in the cross-section of the liquid sheet. The TO-2 refinement ensures that there are at least two cells in the thickness direction in spite of the dynamic variations of the sheet thickness and shape.

The jet dynamics and subsequent atomization can be characterized by two non-dimensional parameters: the Weber and Reynolds numbers. They are defined respectively as We $=\rho u_{j}^{2} D / \sigma$, and, $\operatorname{Re}=\rho u_{j} D / \mu$, where $\rho$ is the liquid density, $D$ the jet diameter, $u_{j}$ the mean jet velocity, $\sigma$ the surface tension, and $\mu$ the viscosity of the liquid. The size of the computational domain is set to be $50 D \times 30 D \times 10 D$, to eliminate the effect of domain boundaries. Standard outflow condition is set to the domain boundaries. The velocity profiles of the liquid jets are set to be parabolic when $\operatorname{Re} \leqslant 4000$ and $1 / 7$ power-law when $\mathrm{Re}>4000$. Gradient-based, curvature-based, and topology-oriented refinements are used concurrently to ensure numerical accuracy and robustness.

Fig. 23 shows the various flow patterns observed at different jet velocities varying from 2.2 to $11.2 \mathrm{~m} / \mathrm{s}$. The different flow patterns are dependent on the Reynolds and Weber numbers. Movies showing the evolution of the liquid rim, sheet, and atomization can be found in Chen and Yang [29]. Fig. 23(a) shows a well-defined fluid chain [30] structure at $u_{j}=$ $2.2 \mathrm{~m} / \mathrm{s}$. Both a liquid sheet and a closed rim form in the first ring. No sheet, however, exists after the second chain link. The colliding streams from the two sides of the rim coalesce into a single column oscillating under the inertial-capillary interaction. Finally, breakup takes place at the end of the column. As the jet velocity increases, a larger liquid sheet is established, as shown in Fig. 23(b). Since the sheet and rim formed afterwards are much smaller, this pattern is usually referred to as a closed rim. Fig. 23(c) shows a pattern known as a disintegrating sheet [30]. The thin liquid sheet in the downstream region is prone to small disturbances. Holes are formed and grow progressively, eventually reaching the sheet boundary to open the rim. The ligaments downstream of the opening break up because of capillary action. As the jet velocity further increases to $7.9 \mathrm{~m} / \mathrm{s}$, the liquid rim becomes unstable, as shown in Fig. 23(d). Disturbances originate at the impingement point and grow, while propagating downstream along the liquid rim. Ligaments are then formed, and break 
up into droplets due to the end-pinching mechanism. In the extreme case of $u_{j}=11.2 \mathrm{~m} / \mathrm{s}$, shown in Fig. 23(e), the liquid sheet becomes unstable, with violent flapping, followed by rapid atomization into droplets.

\section{Conclusions and remarks}

Two thickness-based (distance- and topology-oriented) adaptive mesh refinement schemes were developed to resolve thin regions in multiphase flowfield efficiently and automatically. Both schemes were incorporated in a general framework with a volume-of-fluid formulation. A distance-oriented criterion was proposed for thin regions near fixed boundaries, while a topology-oriented criterion can be used in arbitrary conditions. Implementations of these thickness-based refinement schemes are straightforward, requiring only a few steps to locate coarse cells. Numerical experiments were conducted to validate the capabilities of the proposed algorithms. These included the dynamics of colliding droplets, droplet motions in a microchannel, and atomization of liquid impinging jets.

The thickness-based refinement methods developed in the present study are efficient and robust. The thin regions can be detected and refined swiftly and dynamically through simple logical judgments. More importantly, no thickness information, equation solving, variable averaging or mesh repairing is needed. It should be noted that the mesh refinement techniques presented here can be extended to problems other than multi-phase flows through selection of appropriate variables to represent the thin regions.

\section{Acknowledgements}

This work was financially supported by the Army Research Office under the Multi-University Research Initiative under Contract No. W911NF-08-1-0124. The support and encouragement provided by Dr. Ralph Anthenien are gratefully acknowledged. Special thanks is due to Dr. Stéphane Popinet for allowing us to use his work on the VOF and AMR algorithms in the Gerris code.

\section{References}

[1] M.J. Berger, P. Colella, Local adaptive mesh refinement for shock hydrodynamics, J. Comput. Phys. 82 (1989) 64-84.

[2] A.M. Khokhlov, Fully threaded tree algorithms for adaptive refinement fluid dynamics simulations, J. Comput. Phys. 143 (1998) $519-543$.

[3] X. Zheng, J. Lowengrub, A. Anderson, V. Cristini, Adaptive unstructured volume remeshing-II: Application to two-and three-dimensional level-set simulations of multiphase flow, J. Comput. Phys. 208 (2005) 626-650.

[4] H.D. Ceniceros, A.M. Roma, A. Silveira-Neto, M.M. Villar, A robust, fully adaptive hybrid level-set/front-tracking method for two-phase flows with an accurate surface tension computation, Commun. Comput. Phys. 8 (2010) 51-94.

[5] M. Malik, E.S.C. Fan, M. Bussmann, Adaptive VOF with curvature-based refinement, Int. J. Numer. Methods Fluids 55 (2007) $693-712$.

[6] M. Dai, D.P. Schmidt, Adaptive tetrahedral meshing in free-surface flow, J. Comput. Phys. 208 (2005) $228-252$.

[7] M. Sussman, A.S. Almgren, J.B. Bell, P. Colella, L.H. Howell, M.L. Welcome, An adaptive level set approach for incompressible two-phase flows, J. Comput. Phys. 148 (1999) 81-124.

[8] S. Popinet, An accurate adaptive solver for surface-tension-driven interfacial flows, J. Comput. Phys. 228 (2009) $5838-5866$.

[9] V. Cristini, J. Bławzdziewicz, M. Loewenberg, An adaptive mesh algorithm for evolving surfaces: simulations of drop breakup and coalescence, J. Comput. Phys. 168 (2001) 445-463.

[10] R.H. Nochetto, S.W. Walker, A hybrid variational front tracking-level set mesh generator for problems exhibiting large deformations and topological changes, J. Comput. Phys. 229 (2010) 6243-6269.

[11] S. Quan, Simulations of multiphase flows with multiple length scales using moving mesh interface tracking with adaptive meshing, J. Comput. Phys, 230 (2011) 5430-5448.

[12] J. Brackbill, D. Kothe, C. Zemach, A continuum method for modeling surface tension, J. Comput. Phys. 100 (1992) 335-354.

[13] S. Popinet, S. Zaleski, A front-tracking algorithm for accurate representation of surface tension, Int. J. Numer. Methods Fluids 30 (1999) $775-793$.

[14] K.F. Tchon, M. Khachan, F. Guibault, R. Camarero, Three-dimensional anisotropic geometric metrics based on local domain curvature and thickness, Comput. Aided Des. 37 (2005) 173-187.

[15] H. Zhang, G. Zhao, Adaptive hexahedral mesh generation based on local domain curvature and thickness using a modified grid-based method, Finite Elem. Anal. Des. 43 (2007) 691-704.

[16] W.J. Rider, D.B. Kothe, Reconstructing volume tracking, J. Comput. Phys. 141 (1998) 112-152.

[17] J.B. Bell, P. Colella, H.M. Glaz, A second-order projection method for the incompressible Navier-Stokes equations, J. Comput. Phys. 85 (1989) $257-283$.

[18] R.J. LeVeque, High-resolution conservative algorithms for advection in incompressible flow, SIAM J. Numer. Anal. 33 (1996) 627-665.

[19] N. Ashgriz, J.Y. Poo, Coalescence and separation in binary collisions of liquid drops, J. Fluid Mech. 221 (1990) 183-204

[20] J. Qian, C.K. Law, Regimes of coalescence and separation in droplet collision, J. Fluid Mech. 331 (1997) 59-80.

[21] M. Nobari, Y. Jan, G. Tryggvason, Head on collision of drops-A numerical investigation, Phys. Fluids 8 (1996) 29.

[22] K.-L. Pan, C.K. Law, B. Zhou, Experimental and mechanistic description of merging and bouncing in head-on binary droplet collision, J. Appl. Phys. 103 (2008) 064901-064911.

[23] J. Qian, C. Law, Regimes of coalescence and separation in droplet collision, J. Fluid Mech. 331 (1997) 59-80.

[24] W. Lee, G. Son, Numerical study of obstacle configuration for droplet splitting in a microchannel, Comput. Fluids 84 (2013) 351-358.

[25] L. Wu, M. Tsutahara, L.S. Kim, M. Ha, Three-dimensional lattice Boltzmann simulations of droplet formation in a cross-junction microchannel, Int. J. Multiph. Flow 34 (2008) 852-864.

[26] M.M. Dupin, I. Halliday, C.M. Care, Simulation of a microfluidic flow-focusing device, Phys. Rev. E 73 (2006) 055701.

[27] N. Bremond, A.R. Thiam, J. Bibette, Decompressing emulsion droplets favors coalescence, Phys. Rev. Lett. 100 (2008) 024501

[28] X. Chen, D. Ma, V. Yang, S. Popinet, High-fidelity simulations of impinging jet atomization, At. Sprays 23 (2013) 1079-1101.

[29] X. Chen, V. Yang, Impinging jet dynamics, arXiv:1210.3892, 2012.

[30] J.W. Bush, A.E. Hasha, On the collision of laminar jets: fluid chains and fishbones, J. Fluid Mech. 511 (2004) $285-310$. 